\title{
DIGITALCOMMONS
}

—@WAYNESTATE-

Wayne State University

2-1-2009

\section{Second-Order Analysis of Polyhedral Systems in Finite and Infinite Dimensions with Applications to Robust Stability of Variational Inequalities}

\author{
René Henrion \\ Weierstrass Institute for Applied Analysis and Stochastics, Berlin, Germany \\ Boris S. Mordukhovich \\ Wayne State University, boris@math.wayne.edu \\ Nguyen Mau Nam \\ The University of Texas-Pan American, Edinburg, TX, nguyenmn@utpa.edu
}

\section{Recommended Citation}

Henrion, René; Mordukhovich, Boris S.; and Nam, Nguyen Mau, "Second-Order Analysis of Polyhedral Systems in Finite and Infinite Dimensions with Applications to Robust Stability of Variational Inequalities" (2009). Mathematics Research Reports. Paper 64.

http://digitalcommons.wayne.edu/math_reports/64

This Technical Report is brought to you for free and open access by the Mathematics at DigitalCommons@WayneState. It has been accepted for inclusion in Mathematics Research Reports by an authorized administrator of DigitalCommons@WayneState. 


\section{SECOND-ORDER ANALYSIS OF POLYHEDRAL SYSTEMS IN FINITE AND INFINITE DIMENSIONS WITH APPLICATIONS TO ROBUST STABILITY OF VARIATIONAL INEQUALITIES}

RENÉ HENRION, BORIS S. MORDUKHOVICH and NGUYEN MAU NAM

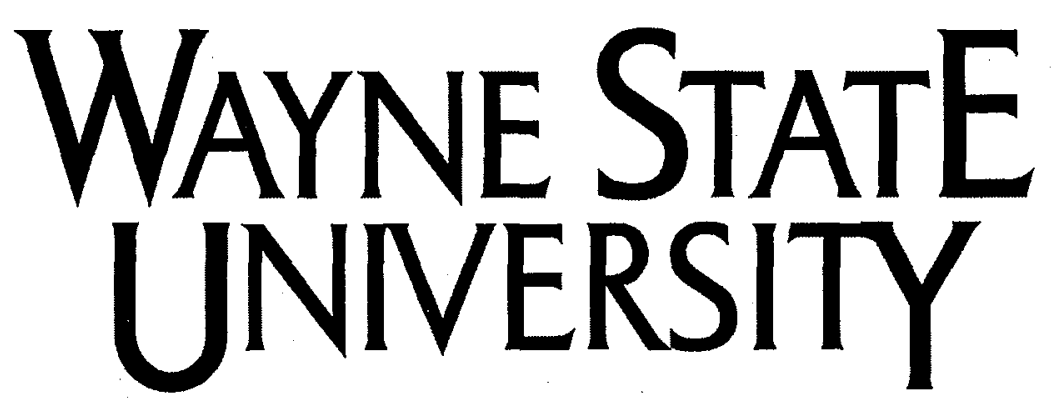

Detroit, MI 48202

Department of Mathematics

Research Report

\section{Series}

\#2 


\title{
SECOND-ORDER ANALYSTS OF POLYHEDRAL SYSTEMS IN FINITE AND INFINITE DIMENSIONS WITH APPLICATIONS TO ROBUST STABILITY OF VARIATIONAL INEQUALITIES
}

\author{
RENÉ HENRION ${ }^{1}$, BORIS S. MORDUKHOVICH ${ }^{2}$ and NGUYEN MAU NAM ${ }^{3}$
}

\begin{abstract}
This paper concerns second-order analysis for a remarkable class of variational systems in finite-dimensional and infinite-dimensional spaces, which is particularly important for the study of optimization and equilibrium problems with equilibrium constraints. Systems of this type are described via variational inequalities over polyhedral convex sets and allow us to provide a comprehensive local analysis by using appropriate generalized differentiation of the normal cone mappings for such sets. In this paper we efficiently compute the required coderivatives of the normal cone mappings exclusively via the initial data of polyhedral sets in reflexive Banach spaces. This provides the main tools of second-order variational analysis allowing us, in particular, to derive necessary and sufficient conditions for robust Lipschitzian stability of solution maps to parameterized variational inequalities with evaluating the exact bound of the corresponding Lipschitzian moduli. The efficient coderivative calculations and characterizations of robust stability obtained in this paper are the first results in the literature for the problems under consideration in infinite-dimensional spaces. Most of them are also new in finite dimensions.

Key words. variational analysis and optimization, reflexive Banach spaces, polyhedral sets, parametric variational inequalities, robust stability, generalized differentiation, coderivatives and second-order subdifferentials
\end{abstract}

AMS subject classification. 49J52, 49K40, 58C20

\section{Introduction}

It has been well recognized in optimization and variational analysis, starting with the seminal work by Robinson [23], that a number of the most interesting variational systems and variational conditions can be described via the normal cone mapping $N(x ; \Theta)$ to convex sets $\Theta \subset X$ as well as their subdifferential counterparts and further nonconvex extensions. Among variational models of this type we mention variational inequalities, complementarity problems, KKT (Karush-Kuhn-Tucker) conditions in parametric optimization, and other variational and equilibrium systems arising in optimization theory and its numerous applications; see, e.g., $[4,9,10,15,16,19,20,23,24,25]$ and the references therein. Particularly important classes of sets used in describing variational and equilibrium conditions can be

\footnotetext{
${ }^{1}$ Weierstrass Institute for Applied Analysis and Stochastics, 10117 Berlin, Germany (henrion@wiasberlin.de). Research of this author was partially supported by the DFG Research Center MATHEON in Berlin.

${ }^{2}$ Department of Mathematics, Wayne State University, Detroit, MI 48202, USA (boris@math.wayne.edu). Research of this author was partially supported by the National Science Foundation under grant DMS0603846.

${ }^{3}$ Department of Mathematics, The University of Texas-Pan American, Edinburg, TX 78539-2999, USA (nguyenmn@utpa.edu). Research of this author was partially supported by the Faculty Research Council at the University of Texas-Pan American.
} 
represented in the following convex polyhedral form

$$
\Theta:=\left\{x \in X \mid\left\langle x_{i}^{*}, x\right\rangle \leq 0, \quad i \in T:=\{1, \ldots, m\}\right\},
$$

where $x_{i}^{*}$ are given elements of the dual space $X^{*}$. Note that the homogeneous/conic form of polyhedral systems in (1.1) does not restrict the generality, since nonhomogeneous polyhedra can always be locally translated to (1.1).

Known results on variational analysis involving the normal cone mappings to convex polyhedra and its efficient implementation for important classes of optimization and equilibrium problems concern the case of finite-dimensional spaces $X=\mathbb{R}^{n}$. The reader can find more information on these and related developments and applications in $[3,4,6,7$, $18,19,25,27,28,29]$ and the references therein. It comes naturally that local variational analysis of the normal cone mapping

$$
\mathcal{F}(x):=N(x ; \Theta), \quad x \in X
$$

associated with (1.1) and its applications to, e.g., deriving optimality and stationarity conditions, sensitivity and stability issues, etc. call for the usage and implementation of appropriate constructions of generalized differentiation for set-valued mappings of type (1.2).

Among other generalized differential constructions, coderivatives of set-valued mappings introduced in [12] have been well recognized as a powerful tool of variational analysis and its numerous applications, particularly to problems of optimization, equilibria, and control; see, e.g., the books $[2,15,16,19,25,26]$ with their references and discussions. To proceed efficiently with applications of coderivatives, we need to compute them constructively in terms of the initial data of the problems in question. Various results in this direction for the normal cone mapping generated by polyhedral sets in finite-dimensional spaces can be found in $[3,6,7,27,28]$. To the best of our knowledge, the constructive results obtained in this paper are the first ones for polyhedral sets in infinite dimensions providing also new developments and applications in finite-dimensional settings.

Observe that coderivatives of the normal cone mapping (1.2) accumulate in fact some second-order information on the original polyhedral set (1.1), which is used in variational analysis of first-order optimality and/or equilibrium conditions (e.g., of the KKT type) exhibited by the normal cone mapping under consideration.

The underlying framework of this paper is the class of reflexive Banach spaces $X$. Our primary goal is to precisely compute the basic/limiting coderivative by Mordukhovich for the normal cone mapping (1.2), which is actually the second-order subdifferential [13] of the indicator function associated with the polyhedral set (1.1); see Remark 4.8 in Section 4 for more details. Then we apply the obtained coderivative formulas to derive efficient conditions for robust Lipschitzian stability of solution maps to parameterized variational inequalities via the coderivative characterization of the major Lipschitz-like/Aubin property for general set-valued mappings between Asplund (in particular, reflexive) spaces, with computing the exact bound of Lipschitzian moduli. As auxiliary results of their own independent interest, we evaluate the so-called precoderivative (known also as the Fréchet coderivative) of the normal cone mapping (1.2) generated by (1.1), which is a crucial building block for computing the basic/limiting coderivative of (1.2) in reflexive Banach spaces. 
Besides employing fundamental tools of variational analysis and generalized differentiation taken mainly from [15], we use in this study an appropriate infinite-dimensional version of the classical Farkas lemma, in the form of Motzkin's theorem of the alternative (see, e.g., [1]), that largely exploits the polyhedral structure of (1.1) described by linear inequalities.

The rest of the paper is organized as follows. Section 2 contains some basic definitions and preliminary material from variational analysis, generalized differentiations, and linear inequalities widely used in formulations and proofs of the main results.

Section 3 deals with computing the prenormal cone (or the Fréchet normal cone) to the graph of (1.2) and the corresponding precoderivative of $\mathcal{F}$ in terms constructively generated by the initial data of the given polyhedral set (1.1). The results obtained are the first ones in this direction for the case of infinite-dimensional spaces being mostly new and/or improving known results of this type in finite dimensions $[3,27]$.

Section 4 is mainly devoted to precise computing, exclusively via the initial data of (1.1), the basic normal cone to the graph of the normal cone mapping (1.2) and the basic coderivative of $\mathcal{F}$ by using, among other devices, the passage to the limit procedures from the corresponding results of Section 3. Furthermore, we show that the basic normal and coderivative constructions are invariant for the normal cone mapping generated by the convex polyhedron under consideration while replacing the weak convergence by the norm convergence on the the space $X$ and its topological dual $X^{*}$. We compare the results obtained here, which are the first in infinite dimensions, with calculating the basic coderivative of $\mathcal{F}$ for convex polyhedral sets given in $[3,7,27]$ in the case of finite-dimensional spaces.

The final Section 5 concerns deriving verifiable conditions for robust Lipschitzian stability of solution maps to parameterized variational inequalities generated by the normal cone mapping to the polyhedral set (1.1) in reflexive Banach spaces. Based on the coderivative characterizations of the Lipschitz-like property for general closed-graph mappings from [15], on some results of coderivative calculus, and largely on the precise computation of the basic coderivative for the normal cone mapping (1.2) given in Section 4, we establish constructive criteria as well as easily verifiable sufficient conditions for robust Lipschitzian stability of the solution maps in question expressed exclusively via the initial data of model (1.1) in both finite-dimensional and reflexive Banach spaces. The results obtained, being the first ones in infinite dimensions, are also new in finite-dimensional settings providing characterizations of robust stability of parametric variational inequalities entirely via their initial data and essentially improving the corresponding results of $[3,28]$. Moreover, we derive constructive estimates as well as precise equalities, new in both finite and infinite dimensions, for computing the exact Lipschitzian bounds for solution maps to the polyhedral variational inequalities under consideration.

Our notation and terminology are basically standard and conventional in the area of variational analysis and generalized differentiation; see, e.g., $[15,25,26]$. Although most of the definitions and some results hold in more general Banach space settings, our standing assumption in this paper (unless otherwise stated) is that the Banach space $X$ in question is reflexive, since the reflexivity seems to be essential for the validity of the main results obtained below. As usual, $\|\cdot\|$ stands for the norm on $X,\langle\cdot, \cdot\rangle$ stands for the canonical pairing between $X$ and its topologically dual space $X^{*}$, the symbol $x_{k}^{*} \stackrel{w}{\rightarrow} x^{*}$ with $k \in \mathbb{N}:=$ 
$\{1,2, \ldots\}$ indicates the weak convergence of a sequence in $X^{*}$. We use the generic symbol * to signify duality/polarity relationships if no confusion arises. In particular,

$$
K^{*}:=\left\{x^{*} \in X^{*} \mid\left\langle x^{*}, x\right\rangle \leq 0 \text { for all } x \in K\right\}
$$

is the polar cone to a cone $K \subset X$. By

$$
\operatorname{ker}\left\{v_{j}^{*} \mid j \in J\right\}:=\left\{x \in X \mid\left\langle v_{j}^{*}, x\right\rangle=0 \text { for all } j \in J\right\}
$$

we denote the kerner/orthogonality subspace generated by the elements $v_{j}^{*} \in X^{*}, j \in J$. In the case of just one generating element $v^{*} \in X^{*}$, we also use the notation

$$
\left\{v^{*}\right\}^{\perp}:=\left\{x \in X \mid\left\langle v^{*}, x\right\rangle=0\right\} .
$$

The notation $A X$ stands for the image/range subspace of the linear operator $A: X \rightarrow Y$.

Given further a nonempty set $\Omega \subset X$, denote by $\operatorname{span} \Omega$ the smallest linear subspace containing $\Omega$ and by cone $\Omega$ the smallest convex cone containing this set; by convention we let cone $\emptyset:=\{0\}$ and span $\emptyset:=\{0\}$. The $\Omega$-restricted convergence $x \stackrel{\Omega}{\rightarrow} \bar{x}$ means that $x \rightarrow \bar{x}$ with $x \in \Omega$. Considering finally a set-valued mapping $F: X \rightrightarrows X^{*}$, define its domain by

$$
\operatorname{Dom} F:=\{x \in X \mid F(x) \neq \emptyset\}
$$

and the (sequential) Painlevé-Kuratowski outer/upper limit of $F$ as $x \rightarrow \bar{x}$ by

$$
\begin{aligned}
\operatorname{Limsup}_{x \rightarrow \bar{x}} F(x):=\left\{x^{*} \in X^{*} \mid\right. & \exists \text { sequences } x_{k} \rightarrow \vec{x}, x_{k}^{*} \stackrel{w}{\rightarrow} x^{*} \text { as } k \rightarrow \infty \\
& \text { with } \left.x_{k}^{*} \in F\left(x_{k}\right) \text { for all } k \in \mathbb{N}\right\} .
\end{aligned}
$$

\section{Basic Definitions and Preliminaries}

In our brief descriptions of basic tools and preliminary results of variational analysis and generalized differentiation presented in this section we follow the book [15], where more details, proofs, and discussions can be found. We also refer the reader to $[2,16,26,25]$ for related and additional material.

As mentioned in Section 1, our underlying assumption is that all the spaces in question are Banach and reflexive, which is the standing setting of this paper unless otherwise stated. Note that any reflexive Banach space is Asplund, and thus the major results from [15] established in Asplund spaces are applied in the setting of this paper. In [15] the reader can find appropriate counterparts of the basic definitions and results presented in this section in more general settings of Asplund spaces and also of arbitrary Banach spaces.

Given a nonempty set $\Omega \subset X$, define the prenormal cone (known also as the Fréchet or regular normal cone) to $\Omega$ at $\bar{x} \in \Omega$ by

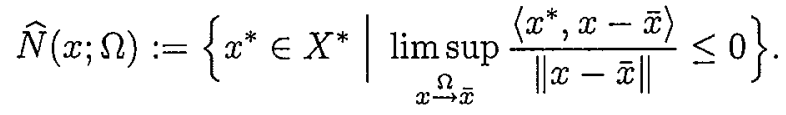

For convenience let $\widehat{N}(x ; \Omega)=\emptyset$ if $x \notin \Omega$. Note that the set $\widehat{N}(\bar{x} ; \Omega)$ is convex and weakly closed in $X^{*}$; furthermore, it reduces to the normal cone of convex analysis if $\Omega$ is convex. 
However, the prenormal cone $(2.1)$ may be trivial $(=\{0\})$ at boundary points of simple nonconvex sets in $\mathbb{R}^{2}$ (see examples in $[15,25]$ ), and it does not generally admit pointwise calculus (e.g., the crucial intersection rule) required by many applications. The situation is dramatically improved when we consider the sequential regularization of (2.1) employing the outer limit $(1.3)$ to $\widehat{N}(\cdot ; \Omega)$ by

$$
N(\bar{x} ; \Omega):=\operatorname{Limsup}_{x \rightarrow \bar{x}} \hat{N}(x ; \Omega)
$$

known as the (basic, limiting, Mordukhovich) normal cone to $\Omega$ at $\bar{x} \in \Omega$. When $X=\mathbb{R}^{n}$, construction (2.2) can be equivalently described in the form:

$$
\begin{aligned}
N(\bar{x} ; \Omega)=\left\{x^{*} \in \mathbb{R}^{n} \mid\right. & \text { there exist } \alpha_{k} \geq 0, x_{k} \rightarrow \bar{x}, w_{k} \in \Omega \text { for } k \in \mathbb{N} \text { such that } \\
& \left.\left\|w_{k}-x_{k}\right\|=\operatorname{dist}\left(x_{k} ; \Omega\right) \text { and } \alpha_{k}\left(x_{k}-w_{k}\right) \rightarrow x^{*} \text { as } k \rightarrow \infty\right\}
\end{aligned}
$$

originally introduced in [11] via the Euclidean distance function dist $(x ; \Omega)$ to $\Omega$. Observe that the basic normal cone (2.2) is often nonconvex in the case of nonconvex sets $\Omega$ while it and the corresponding subdifferential/coderivative constructions enjoy full calculus and other important properties required by applications. These developments are mainly based on variational/extremal principles of variational analysis, which replace the classical convex separation theorems in nonconvex settings.

Given next a set-valued mapping $F: X \rightrightarrows Y$ with the graph

$$
\operatorname{gph} F:=\{(x, y) \in X \times Y \mid y \in F(x)\}
$$

and following the pattern initiated in [12], we present two constructions of coderivative type via the corresponding normals to the graphical set (2.3). The precoderivative (or Fréchet coderivative) of $F$ at $(\bar{x}, \bar{y}) \in \mathrm{gph} F$ is defined by

$$
\widehat{D}^{*} F(\bar{x}, \bar{y})\left(y^{*}\right):=\left\{x^{*} \in X^{*} \mid\left(x^{*},-y^{*}\right) \in \widehat{N}((\bar{x}, \bar{y}) ; \operatorname{gph} F)\right\}, \quad y^{*} \in Y^{*},
$$

while the basic/limiting coderivative of $F$ at $(\bar{x}, \bar{y})$ is

$$
D^{*} F(\bar{x}, \bar{y})\left(y^{*}\right):=\left\{x^{*} \in X^{*} \mid\left(x^{*},-y^{*}\right) \in N((\bar{x}, \bar{y}) ; \operatorname{gph} F)\right\}, \quad y^{*} \in Y^{*},
$$

which corresponds to the "normal" coderivative construction in [15]. If the given mapping $F=f: X \rightarrow Y$ is single-valued and strictly differentiable at $\bar{x}$ with the derivative $\nabla f(\bar{x}): X \rightarrow Y$, in the sense that

$$
\lim _{x, u \rightarrow \bar{x}} \frac{f(x)-f(u)-\langle\nabla f(\bar{x}), x-u\rangle}{\|x-u\|}=0
$$

(this is automatic when $f$ is $C^{1}$ around $\bar{x}$ ), then

$$
\widehat{D}^{*} f(\bar{x})\left(y^{*}\right)=D^{*} f(\bar{x})\left(y^{*}\right)=\left\{\nabla f(\bar{x})^{*} y^{*}\right\} \text { for all } y^{*} \in Y^{*}
$$

where $\bar{y}=f(\bar{x})$ is omitted in the coderivative notation for single-valued mappings. The coderivative representations in (2.7) show that both constructions (2.4) and (2.5) reduce to the adjoint derivative operator in the classical setting. 
It is easily implied by the definitions that the basic coderivative (2.5) admits the following limiting representation via the precoderivative (2.4) at points thereby:

$$
D^{*} F(\bar{x}, \bar{y})\left(y^{*}\right)=\operatorname{Limsup}_{\substack{(x, y) \rightarrow(\bar{x}, \bar{y}) \\ z^{*} \stackrel{w}{\rightarrow} y^{*}}} \widehat{D}^{*} F(x, y)\left(z^{*}\right),
$$

where the outer limit (1.3) is taken with respect to the weak topology in both dual spaces $X^{*}$ and $Y^{*}$. We say that $F$ is (strongly) coderivatively normal at $(\bar{x}, \bar{y})$ if

$$
D^{*} F(\bar{x}, \bar{y})\left(y^{*}\right)=\operatorname{Limsup}_{\substack{(x, y) \rightarrow(\bar{x}, \bar{y}) \\\left\|z^{*}-y^{*}\right\| \rightarrow 0}} \widehat{D}^{*} F(x, y)\left(z^{*}\right),
$$

which means that the coderivative construction (2.5) does not change if we replace the weak convergence $z^{*} \stackrel{w}{\rightarrow} y^{*}$ in (2.8) by the norm one $z^{*} \rightarrow y^{*}$ in (2.9), while the convergence on $X^{*}$ in (2.9) stays weak by (1.3). Note that the right-hand side limit in (2.9) corresponds to the "mixed" coderivative construction in [15]. We refer the reader to Proposition 4.9 in [15] that lists a number of efficient conditions ensuring the coderivative normality of set-valued and single-valued mappings. Standard classes of mappings satisfying (2.9) include of course those with convex graph (2.3) as well as strictly differentiable (2.6) at the point in question.

Recall also a certain. "normal compactness" property of set-valued mappings that is needed for characterizing robust Lipschitzian stability in infinite dimensions. A mapping $F: X \rightrightarrows Y$ is partially sequentially normally compact (PSNC) at $(\bar{x}, \bar{y}) \in \operatorname{gph} F$ if for any sequence $\left\{\left(x_{k}, y_{k}, x_{k}^{*}, y_{k}^{*}\right)\right\} \subset X \times Y \times X^{*} \times Y^{*}$ satisfying $\left(x_{k}^{*}, y_{k}^{*}\right) \in \widehat{N}\left(\left(x_{k}, y_{k}\right) ; \operatorname{gph} F\right)$ for all $k \in \mathbb{I N}$ we have the implication

$$
\left[\left(x_{k}, y_{k}\right) \rightarrow(\bar{x}, \bar{y}), \quad x_{k}^{*} \stackrel{w}{\rightarrow} 0, \quad\left\|y_{k}^{*}\right\| \rightarrow 0\right] \Longrightarrow\left\|x_{k}^{*}\right\| \rightarrow 0 \text { as } k \rightarrow \infty .
$$

The PSNC property obviously holds if the domain space $X$ is finite-dimensional. In fact, it holds in much more general settings of infinite-dimensional spaces being stable with respect to various operations performed on set-valued and single-valued mappings; the latter calculus based on the extremal/variational principles can be found in [15]. In particular, $F$ is PSNC at $(\vec{x}, \bar{y})$ if it is Lipschitz-like around this point, i.e., there are neighborhoods $U$. of $\bar{x}$ and $V$ of $\bar{y}$ such that

$$
F(x) \cap V \subset F(u)+\ell\|x-u\| \mathbb{B} \text { whenever } x, u \in U
$$

with some constant/modulus $\ell \geq 0$, where $\mathbb{B}$ stands for the closed unit ball in the space in question. The infimum of all moduli $\{\ell\}$ in (2.11) is called the exact Lipschitzian bound of $F$ around $(\bar{x}, \bar{y})$ and is denoted by $\operatorname{lip} F(\bar{x}, \bar{y})$. Note that property $(2.11)$ seems to be the most natural extension of the classical (robust) local Lipschitzian behavior to set-valued mappings. It is also known as Aubin's "pseudo-Lipschitzian" property and reduces to the Hausdorff one around $\bar{x}$ for $V=Y$ in (2.11). It has been well recognized and employed in variational analysis that the robust Lipschitzian property $(2.11)$ is equivalent to metric regularity and linear openness of the inverse mapping $F^{-1}$; see, e.g., $[8,15,25]$.

The following coderivative characterization of the Lipschitz-like property (2.11) as well as a lower estimate and the precise formula for computing the exact bound of Lipschitzian moduli in (2.11) are consequences of Theorem 4.10 from [15], where the reader can find more general results, discussions, and references. 
Theorem 2.1 (coderivative characterization and exact bound formula for Lipschitzlike mappings). Let $F: X \rightrightarrows Y$ be closed-graph around $(\bar{x}, \bar{y}) \in \operatorname{gph} F$ and coderivatively normal at this point. Then $F$ is Lipschitz-like around $(\bar{x}, \bar{y})$ if and only if

$$
D^{*} F(\bar{x}, \bar{y})(0)=\{0\}
$$

and $F$ is PSNC at $(\bar{x}, \bar{y})$. Furthermore, we have the estimate

$$
\operatorname{lip} F(\bar{x}, \bar{y}) \geq\left\|D^{*} F(\bar{x}, \bar{y})\right\|:=\sup \left\{\left\|x^{*}\right\| \mid x^{*} \in D^{*} F(\bar{x}, \bar{y})\left(y^{*}\right),\left\|y^{*}\right\| \leq 1\right\},
$$

which holds as equality if $\operatorname{dim} X<\infty$.

When both $X$ and $Y$ are finite-dimensional, the results of Theorem 2.1 reduce to those obtained in [14]; see also [25, Theorem 9.40] and the references therein.

Finally in this section, we present an appropriate infinite-dimensional version of generalized Farkas lemma, in the form of Motzkin's theorem of the alternative, which is taken from $[1$, Theorem 5$]$ and is widely used in the paper.

Theorem 2.2 (of the alternative). Let $W$ be a vector space of arbitrary dimension, and let $A: W \rightarrow \mathbb{R}^{d}$ and $B: W \rightarrow \mathbb{R}^{s}$ be linear mappings. Then we have the alternative:

$$
\begin{aligned}
& \text { either: } \exists x \in W \text { such that } B x \geq 0 \text { and } A x>0 \text { componentwise, } \\
& \text { or: } \quad \exists \lambda \in \mathbb{R}^{d}, \exists \mu \in \mathbb{R}^{s} \text { such that } \lambda \geq 0, \lambda \neq 0, \mu \geq 0, \text { and } \\
& \\
& \sum_{i=1}^{d} \lambda_{i} A_{i}+\sum_{j=1}^{s} \mu_{j} B_{j}=0,
\end{aligned}
$$

where $A_{i}$ and $B_{j}$ refer to the components of $A$ and $B$, respectively.

\section{Computing Precoderivatives of Normal Cone Mappings to Convex Polyhedra}

Given the index $\operatorname{set} T=\{1, \ldots, m\}$ as $m \geq 1$ and the generating linear functionals $x_{i}^{*} \in X^{*}$, $i \in T$, we rewrite the initial convex polyhedron (1.1) as

$$
\Theta=\left\{x \in X \mid\left\langle x_{i}^{*}, x\right\rangle \leq 0 \text { for all } i \in T\right\}
$$

and fix some point $\vec{x} \in \Theta$. Consider the normal cone of convex analysis

$$
N(\bar{x} ; \Theta):=\left\{x^{*} \in X \mid\left\langle x^{*}, x-\bar{x}\right\rangle \leq 0 \text { for all } x \in \Theta\right\}
$$

to $\Theta$ at $\bar{x} \in \Theta$ and define the collection of active constraint indices

$$
I(\bar{x}):=\left\{i \in T \mid\left\langle x_{i}^{*}, \bar{x}\right\rangle=0\right\},
$$

where the dependence on $\bar{x}$ in notation (3.3) may be omitted if no confusion arises.

Our main goal in this section is to provide an exact calculation of the prenormal cone (2.1) to the (nonconvex) graph of the normal cone mapping $\mathcal{F}$ in (1.2) generated by (3.2) 
and hence the precoderivative (2.4) of the mapping $\mathcal{F}$ entirely in terms of the initial data of (3.1) including the active constraint indices (3.3) at the reference point $\bar{x}$.

To proceed, recall the standard construction of the tangent cone

$$
T(\bar{x} ; \Theta):=N^{*}(\bar{x} ; \Theta)=\left\{v \in X \mid\left\langle v, x^{*}\right\rangle \leq 0 \text { for all } x^{*} \in N(\bar{x} ; \Theta)\right\}
$$

to the convex set $\Theta$ at $\bar{x}$ defined as the dual/polar cone to the normal one (3.2) in the case of reflexive spaces under consideration.

In our polyhedral case (3.1) the normal and tangent cones to $\Theta$ admit the following explicit representations (probably well known while we did no find the exact references) via the generating elements $x_{i}^{*}$ in (3.1) and the active indices $I(\bar{x})$.

Proposition 3.1 (explicit representations of the normal and tangent cones to convex polyhedra). Let $\Theta$ be given in (3.1), and let $I(\bar{x})$ be defined in (3.3). Then we have the representations

$$
\begin{gathered}
N(\bar{x} ; \Theta)=\text { cone }\left\{x_{i}^{*} \mid i \in I(\bar{x})\right\}=\left\{\sum_{i \in I(\bar{x})} \lambda_{i} x_{i}^{*} \mid \lambda_{i} \geq 0\right\}, \\
T(\bar{x} ; \Theta)=\left\{v \in X \mid\left\langle x_{i}^{*}, v\right\rangle \leq 0 \text { for all } i \in I(\bar{x})\right\} .
\end{gathered}
$$

Proof. The first representation of the normal cone in (3.5) can be easily derived from Theorem 2.2 of the alternative. The second one in (3.5) follows from the first equality in therein by the definition of the conic convex hull in the case of the finite set $\left\{x_{i}^{*} \mid i \in I(\bar{x})\right\}$. The tangent cone representation (3.6) is a direct consequence of the first equality in (3.5) and definition (3.4) of the tangent cone.

Now we consider the set-valued normal cone mapping $\mathcal{F}: X \rightrightarrows X^{*}$ defined in (1.2) by $\mathcal{F}(x)=N(x ; \Theta)$ and establish an intermediate representation of the prenormal cone (2.1) to the graph of $\mathcal{F}$ at $\left(\bar{x}, \bar{x}^{*}\right) \in \operatorname{gph} \mathcal{F}$ with some $\bar{x}^{*} \in N(\bar{x} ; \Theta)$ via the tangent cone (3.4) to the original polyhedral set (3.1) at the reference point $\bar{x}$, which essentially exploits the reflexivity of the space $X$.

Proposition 3.2 (tangent representation of the prenormal cone to the graph of the normal cone mapping). Fix $\bar{x}^{*} \in N(\bar{x} ; \Theta)$ from the normal cone $(3.2)$ to the polyhedral set (3.1). Then we have the following representation of the prenormal cone (2.1) to the graph of the normal cone mapping (1.2):

$$
\widehat{N}\left(\left(\bar{x}, \bar{x}^{*}\right) ; \operatorname{gph} \mathcal{F}\right)=\left(T(\bar{x} ; \Theta) \cap\left\{\bar{x}^{*}\right\}^{\perp}\right)^{*} \times\left(T(\bar{x} ; \Theta) \cap\left\{\bar{x}^{*}\right\}^{\perp}\right)
$$

Proof. Take $\bar{x}^{*} \in N(\bar{x} ; \Theta)$ and fix an arbitrary pair $\left(x^{*}, u\right) \in \hat{N}\left(\left(\bar{x}, \bar{x}^{*}\right) ; \operatorname{gph} \mathcal{F}\right)$. It follows from definition (2.1) of the prenormal cone to the graph of $\mathcal{F}$ that

$$
\limsup _{\left(x, u^{*}\right)^{\operatorname{ghp} \mathcal{F}} \rightarrow\left(\bar{x}, \bar{x}^{*}\right)} \frac{\left\langle x^{*}, x-\bar{x}\right\rangle+\left\langle u, u^{*}-\bar{x}^{*}\right\rangle}{\|x-\bar{x}\|+\left\|u^{*}-\bar{x}^{*}\right\|} \leq 0 .
$$


Substituting $x=\bar{x}$ and $u^{*} \in \mathcal{F}(\bar{x})$ into (3.8) and using the fact that the set $\mathcal{F}(\bar{x})$ is a convex cone and then construction (3.4) of the tangent cone to $\Theta$ at $\bar{x}$, we deduce from (3.8) that

$$
u \in \widehat{N}\left(\bar{x}^{*} ; \mathcal{F}(\bar{x})\right)=N\left(\bar{x}^{*} ; \mathcal{F}(\bar{x})\right)=\mathcal{F}(\bar{x})^{*} \cap\left\{\bar{x}^{*}\right\}^{\perp}=T(\bar{x} ; \Theta) \cap\left\{\bar{x}^{*}\right\}^{\perp} .
$$

To verify the inclusion " $\subset$ " in (3.7), it remains to check that $x^{*} \in\left(T(\bar{x} ; \Theta) \cap\left\{\bar{x}^{*}\right\}^{\perp}\right)^{*}$, which means the fulfillment of the relationship

$$
\left\langle x^{*}, v\right\rangle \leq 0 \text { for any } v \in T(\bar{x} ; \Theta) \cap\left\{\bar{x}^{*}\right\}^{\perp} .
$$

To proceed, take any $v \in T(\bar{x} ; \Theta) \cap\left\{\bar{x}^{*}\right\}^{\perp}$ and construct the sequence $x_{k}:=\bar{x}+k^{-1} v$ as $k \in \mathbb{N}$. Observe that for the generating elements $x_{i}^{*}$ in (3.1) we have

$$
\left\langle x_{i}^{*}, x_{k}\right\rangle=\left\langle x_{i}^{*}, \bar{x}\right\rangle+k^{-1}\left\langle x_{i}^{*}, v\right\rangle=k^{-1}\left\langle x_{i}^{*}, v\right\rangle \leq 0 \text { whenever } i \in I(\bar{x}) \text { and } k \in \mathbb{N},
$$

since $x_{i}^{*} \in N(\bar{x} ; \Theta)$ for all $i \in I(\bar{x})$ by (3.2) and (3.3). This implies that $x_{k} \in \Theta$ for all $k \in \mathbb{N}$ sufficiently large, since $\left\langle x_{i}^{*}, \bar{x}\right\rangle<0$ as $i \in T \backslash I(\bar{x})$. Furthermore, taking into account that $\bar{x}^{*} \in N(\bar{x} ; \Theta)$ and $v \in\left\{\bar{x}^{*}\right\}^{\perp}$, we get

$$
\left\langle\bar{x}^{*}, x-x_{k}\right\rangle=\left\langle\bar{x}^{*}, x-\bar{x}\right\rangle-k^{-1}\left\langle\bar{x}^{*}, v\right\rangle=\left\langle\bar{x}^{*}, x-\bar{x}\right\rangle \leq 0 \text { for all } x \in \Theta,
$$

which yields, by the construction of $\mathcal{F}$ in $(1.2)$, that $\bar{x}^{*} \in \mathcal{F}\left(x_{k}\right)$, i.e., $\left(x_{k}, \bar{x}^{*}\right) \in \operatorname{gph} \mathcal{F}$ when $k \in \mathbb{N}$ is sufficiently large. It is obvious that $\left(x_{k}, \bar{x}^{*}\right) \rightarrow\left(\bar{x}, \bar{x}^{*}\right)$ as $k \rightarrow \infty$. Putting $\left(x_{k}, \bar{x}^{*}\right)$ for $\left(x, u^{*}\right)$ in (3.8), we conclude by passing to the limit as $k \rightarrow \infty$ that $\left\langle x^{*}, v\right\rangle \leq 0$ and thus arrive at (3.10). Unifying (3.9) and (3.10) allows us to justify the inclusion " $\subset$ " in (3.7).

To prove the opposite inclusion in (3.7), assume by contradiction that there is a pair $\left(x^{*}, v\right) \in X^{*} \times X$ satisfying the relationships

$$
x^{*} \in\left(T(\bar{x} ; \Theta) \cap\left\{\bar{x}^{*}\right\}^{\perp}\right)^{*}, \quad v \in T(\bar{x} ; \Theta) \cap\left\{\bar{x}^{*}\right\}^{\perp}, \quad\left(x^{*}, v\right) \notin \widehat{N}\left(\left(\bar{x}, \bar{x}^{*}\right) ; \operatorname{gph} \mathcal{F}\right) .
$$

The last one in (3.11) ensures, by the structures of the mapping $\mathcal{F}$ and the prenormal cone (2.1) to its graph, the existence of a number $\gamma>0$ and a sequence $\left(x_{k}, v_{k}^{*}\right) \rightarrow\left(\bar{x}, \bar{x}^{*}\right)$ as $k \rightarrow \infty$ such that $x_{k} \in \Theta, v_{k}^{*} \in N\left(x_{k} ; \Theta\right)$, and

$$
\frac{\left\langle x^{*}, x_{k}-\bar{x}\right\rangle+\left\langle v, v_{k}^{*}-\bar{x}^{*}\right\rangle}{\left\|x_{k}-\bar{x}\right\|+\left\|v_{k}^{*}-\bar{x}^{*}\right\|}>\gamma
$$

for all $k \in \mathbb{N}$ sufficiently large. Considering by (3.3) the collection of active constraint indices $I\left(x_{k}\right)$ at $x_{k}$, we can assume by passing to a subsequence if necessary that there is a constant index set $\widetilde{I}$ such that $I\left(x_{k}\right)=\widetilde{I}$ for all $k \in \mathbb{N}$. It easily follows that $\widetilde{I} \subset I(\bar{x})$, since $x_{k} \rightarrow \bar{x}$ as $k \rightarrow \infty$. Taking this into account and employing the normal cone representation (3.5) from Proposition 3.1, we get

$$
v_{k}^{*}=\sum_{i \in I(\bar{x})} \lambda_{i k} x_{i}^{*} \text { with } \lambda_{i k} \geq 0 \text { for all } i \in I(\bar{x}) \text { and } k \in \mathbb{N} \text {. }
$$

The latter implies, by $v \in T(\bar{x} ; \Theta) \cap\left\{\bar{x}^{*}\right\}^{\perp}$ due to the second inclusion in (3.11) and by $x_{i}^{*} \in N(\bar{x} ; \Theta)$ for all $i \in I(\bar{x})$, that

$$
\left\langle v, v_{k}^{*}-\bar{x}^{*}\right\rangle=\left\langle v, v_{k}^{*}\right\rangle=\sum_{i \in I(\bar{x})} \lambda_{i k}\left\langle v, x_{i}^{*}\right\rangle \leq 0, \quad k \in \mathbb{N}
$$


which ensures, in particular, that $x_{k} \neq \bar{x}$ for all $k \in \mathbb{N}$ due to (3.12). By the reflexivity of $X$ and the weak sequential compactness of the unit ball in $X$ we conclude with no loss of generality that there is $z \in X$ with $\|z\| \leq 1$ such that

$$
\frac{x_{k}-\bar{x}}{\left\|x_{k}-\bar{x}\right\|} \stackrel{w}{\rightarrow} z \text { as } k \rightarrow \infty
$$

Since $x_{k} \in \Theta$, it follows from (3.2) that

$$
\left\langle\frac{x_{k}-\bar{x}}{\left\|x_{k}-\bar{x}\right\|}, z^{*}\right\rangle \leq 0 \text { for all } z^{*} \in N(\bar{x} ; \Theta), \quad k \in \mathbb{N},
$$

which implies by passing to the limit as $k \rightarrow \infty$ that $\left\langle z, z^{*}\right\rangle \leq 0$ for all $z^{*} \in N(\bar{x} ; \Theta)$ and hence $z \in T(\bar{x} ; \Theta)$ by (3.4). Thus $\left\langle z, \bar{x}^{*}\right\rangle \leq 0$, since $\bar{x}^{*} \in N(\bar{x} ; \Theta)$. Moreover, it follows from $v_{k}^{*} \in N\left(x_{k} ; \Theta\right)$ and the normal cone definition that

$$
\left\langle v_{k}^{*}, \frac{\bar{x}-x_{k}}{\left\|\bar{x}-x_{k}\right\|}\right\rangle \leq 0 \text { for all } k \in \mathbb{N} .
$$

Passing to the limit in the latter inequality and taking into account that $v_{k}^{*} \rightarrow \bar{x}^{*}$ strongly in $X^{*}$ as $k \rightarrow \infty$, we arrive at $\left\langle\bar{x}^{*},-z\right\rangle \leq 0$ and conclude therefore that $\left\langle\bar{x}^{*}, z\right\rangle=0$, since the opposite inequality was proved above. This gives $z \in\left\{\bar{x}^{*}\right\}^{\perp}$, and hence we get $z \in T(\bar{x} ; \Theta) \cap\left\{\bar{x}^{*}\right\}^{\perp}$. Furthermore, it follows from (3.12) and (3.13) that

$$
\begin{aligned}
\gamma & <\frac{\left\langle x^{*}, x_{k}-\bar{x}\right\rangle+\left\langle v, v_{k}^{*}-\bar{x}^{*}\right\rangle}{\left\|x_{k}-\bar{x}\right\|+\left\|v_{k}^{*}-\bar{x}^{*}\right\|} \\
& \leq \max \left\{0,\left\langle x^{*}, \frac{x_{k}-\bar{x}}{\left\|x_{k}-\bar{x}\right\|}\right\rangle\right\}+\max \left\{0,\left\langle v, \frac{v_{k}^{*}-\bar{x}^{*}}{\left\|v_{k}^{*}-\bar{x}^{*}\right\|}\right\rangle\right\} \\
& \leq \max \left\{0,\left\langle x^{*}, \frac{x_{k}-\bar{x}}{\left\|x_{k}-\bar{x}\right\|}\right\rangle\right\} \text { for all } k \in \mathbb{I}
\end{aligned}
$$

Letting $k \rightarrow \infty$ at the latter expression and remembering that $x^{*} \in\left(T(\bar{x} ; \Theta) \cap\left\{\bar{x}^{*}\right\}^{\perp}\right)^{*}$ by the first assumption in (3.11) and that $z \in T(\bar{x} ; \Theta) \cap\left\{\bar{x}^{*}\right\}^{\perp}$ as proved above, we arrive at

$$
\gamma \leq \max \left\{0,\left\langle x^{*}, z\right\rangle\right\}=0
$$

which contradicts the fact that $\gamma>0$ in (3.12). This justifies the inclusion " $\supset$ " in (3.7) and thus completes the proof of the proposition.

The result of Proposition 3.2 gives a precise representation of the prenormal cone (2.1) to the graph gph $\mathcal{F}$ of the normal cone mapping (1.2) under consideration, while not explicitly via the original polyhedral set $\Theta$ in (3.1) but involving the tangent cone (3.4) to $\Theta$. Our next goal in this section is to establish an explicit representation of this prenormal cone entirely in terms of the initial data of the convex polyhedron (3.1). To proceed, we introduce the following two sets in spaces $X^{*}$ and $X$, respectively, which are constructed via the generating elements $x_{i}^{*}$ in (3.1) and subsets of the index set $T$ in (3.1). Given arbitrary collections of indices $P \subset Q \subset T$, define the sets

$$
A_{Q, P}:=\operatorname{cone}\left\{x_{i}^{*} \mid i \in Q \backslash P\right\}+\operatorname{span}\left\{x_{i}^{*} \mid i \in P\right\},
$$




$$
B_{Q, P}:=\left\{x \in X \mid\left\langle x_{i}^{*}, x\right\rangle=0 \text { for all } i \in P,\left\langle x_{i}^{*}, x\right\rangle \leq 0 \text { for all } i \in Q \backslash P\right\} .
$$

There is a simple duality/polarity relationship between the above sets used in the proofs of the main result of this section and those in Section 4.

Lemma 3.3 (polarity relationship). Let the sets $A_{Q, P}$ and $B_{Q, P}$ be defined in (3.14) and (3.15), respectively, via the initial data of the convex polyhedron (3.1). Then we have

$$
B_{Q, P}^{*}=A_{Q, P} \text { for any } P \subset Q \subset T \text {. }
$$

Proof. The inclusion $B_{Q, P}^{*} \supset A_{Q, P}$ follows directly from definitions (3.14) and (3.15). To justify the opposite inclusion " $C$ " in (3.16), pick an arbitrary element $x^{*} \in B_{Q, P}^{*}$. Then we have $\left\langle x^{*}, x\right\rangle \leq 0$ for all $x \in B_{Q, P}$, which means that there is no $x \in X$ such that

$$
\begin{aligned}
& \left\langle x^{*}, x\right\rangle>0, \quad\left\langle x_{i}^{*}, x\right\rangle \leq 0, \quad\left\langle-x_{i}^{*}, x\right\rangle \leq 0 \text { for all } i \in P \\
& \text { and }\left\langle x_{i}^{*}, x\right\rangle \leq 0 \text { for all } i \in Q \backslash P .
\end{aligned}
$$

Applying now Theorem 2.2 of the alternative, we find numbers $\lambda>0, \mu_{i} \geq 0$ and $\nu_{i} \geq 0$ as $i \in P$, and $\eta_{i} \geq 0$ as $j \in Q \backslash P$ satisfying the equality

$$
\lambda x^{*}=\sum_{i \in Q \backslash P} \eta_{i} x_{i}^{*}+\sum_{i \in P} \mu_{i} x_{i}^{*}-\sum_{i \in P} \nu_{i} x_{i}^{*} .
$$

The latter immediately implies the relationships

$$
\begin{aligned}
x^{*} & =\sum_{i \in Q \backslash P} \lambda^{-1} \eta_{i} x_{i}^{*}+\sum_{i \in P} \lambda^{-1}\left(\mu_{i}-\nu_{i}\right) x_{i}^{*} \\
& \in \operatorname{cone}\left\{x_{i}^{*} \mid i \in Q \backslash P\right\}+\operatorname{span}\left\{x_{i}^{*} \mid i \in P\right\}=A_{Q, P},
\end{aligned}
$$

which justify the inclusion " $\subset$ " in (3.16) and complete the proof of the lemma.

Now we are ready to establish a constructive representation of the prenormal cone (2.1) to the graph of the normal cone mapping (1.2) entirely in terms of the original polyhedral set (3.1). Namely, given any point $\left(\bar{x}, \bar{x}^{*}\right) \in \operatorname{gph} \mathcal{F}$, we represent $\widehat{N}\left(\left(\bar{x}, \bar{x}^{*}\right) ; \operatorname{gph} \mathcal{F}\right)$ via the sets $A_{Q, P}$ and $B_{Q, P}$ from (3.14) and (3.15), respectively, where the index sets $Q$ and $P$ are fully determined by the pair $\left(\bar{x}, \bar{x}^{*}\right)$. More specifically, by $Q$ we take the active constraint indices $I(\bar{x})$ from (3.3), while the index set of "positive multipliers" $P$ is defined as follows: represent $\bar{x}^{*} \in N(\bar{x} ; \Theta)$ by $(3.5)$ of Lemma 3.1 as

$$
\bar{x}^{*}=\sum_{i \in I(\bar{x})} \lambda_{i} x_{i}^{*} \text { with } \lambda_{i} \geq 0 \text { for all } i \in I(\bar{x})
$$

and take $P=J\left(\bar{x}, \bar{x}^{*}\right) \subset I(\bar{x})$, where the latter index set of positive multipliers is given by

$$
J\left(\bar{x}, \bar{x}^{*}\right):=\left\{i \in I(\bar{x}) \mid \lambda_{i}>0\right\} .
$$

Note that the multipliers $\lambda_{i}$ in representation (3.17) may not uniquely defined unless the active generating elements $\left\{x_{i}^{*} \mid i \in I(\bar{x})\right\}$ of (3.1) are linearly independent. Thus the index set of positive multipliers (3.18) is not necessarily unique. It is easy to observe nevertheless that all the subsequent constructions and results involving $J\left(\bar{x}, \bar{x}^{*}\right)$ are invariant with respect to any choice of the multipliers $\lambda_{i}$ and the index set $J\left(\bar{x}, \bar{x}^{*}\right)$ as above. 
Theorem 3.4 (computing the prenormal cone to the graph of the normal cone mapping). Let $\bar{x}^{*} \in N(\bar{x} ; \Theta)$ for the polyhedral set $\Theta$ in (3.1), let the index sets $I=I(\bar{x})$ and $J=J\left(\bar{x}, \bar{x}^{*}\right)$ be defined by (3.3) and by (3.17) and (3.18), respectively, and let the corresponding sets $A_{I, J}$ and $B_{I, J}$ be given in (3.14) and (3.15). Then the prenormal cone (2.1) to the graph of the normal cone mapping $\mathcal{F}(x)=N(x ; \Theta)$ at $\left(\bar{x}, \bar{x}^{*}\right)$ is computed by

$$
\widehat{N}\left(\left(\bar{x}, \bar{x}^{*}\right) ; \operatorname{gph} \mathcal{F}\right)=A_{I, J} \times B_{I, J}
$$

Proof. To verify (3.19), it remains to show, by Proposition 3.2 and Lemma 3.3, that

$$
T(\bar{x} ; \Theta) \cap\left\{\bar{x}^{*}\right\}^{\perp}=B_{I, J} .
$$

The inclusion " $\supset$ " in (3.20) easily follows from the definition of $B_{I, J}$ in (3.15), the tangent cone representation (3.4) in Proposition 3.1, and the representation of

$$
\bar{x}^{*}=\sum_{i \in J} \lambda_{i} x_{i}^{*} \text { with } \lambda_{i}>0 \text { for all } i \in J=J\left(\bar{x}, \bar{x}^{*}\right)
$$

which is an immediate consequence of (3.17) and (3.18).

To verify the opposite inclusion " $C$ " in (3.20), fix any $v \in T(\bar{x} ; \Theta) \cap\left\{\bar{x}^{*}\right\}^{\perp}$ and get $\left\langle x_{i}^{*}, v\right\rangle \leq 0$ for all $i \in I=I(\bar{x})$ by the tangent cone representation (3.6) from Proposition 3.1. Furthermore, by representation (3.21) of $\bar{x}^{*}$ we have

$$
\left\langle\bar{x}^{*}, v\right\rangle=\sum_{i \in J} \lambda_{i}\left\langle x_{i}^{*}, v\right\rangle=0
$$

which yields $\left\langle x_{i}^{*}, v\right\rangle=0$ for all $i \in J$ by the definition of $J=J\left(\vec{x}, \bar{x}^{*}\right)$ in (3.18). This justifies the inclusion " $C$ " in (3.20) and completes the proof of the lemma.

As a direct consequence of Theorem 3.4, we arrive at precise and constructive computing the precoderivative (2.4) of the normal cone mapping $\mathcal{F}(x)=N(x ; \Theta)$.

Corollary 3.5 (computing the precoderivative of the normal cone mapping). In the notation of Theorem 3.4 we have

$$
\widehat{D}^{*} \mathcal{F}\left(\bar{x}, \bar{x}^{*}\right)(u)=\left\{\begin{array}{l}
\operatorname{cone}\left\{x_{i}^{*} \mid i \in I \backslash J\right\}+\operatorname{span}\left\{x_{i}^{*} \mid i \in J\right\} \\
\text { if }\left\langle x_{i}^{*}, u\right\rangle=0 \text { for } i \in J \text { and }\left\langle x_{i}^{*}, u\right\rangle \geq 0 \text { for } i \in I \backslash J \\
\emptyset \text { for all other } u \in X
\end{array}\right.
$$

Proof. Follows directly from definition (2.4) of the precoderivative and the result of Theorem 3.4 for computing the prenormal cone to the graph of $\mathcal{F}$.

\section{Computing Coderivatives of Normal Cone Mappings to Convex Polyhedra}

The main goal of this section is to efficiently compute the (basic, limiting) coderivative (2.5) of the normal cone mapping $\mathcal{F}$ from(1.2) generated by the polyhedral set (3.1). We provide such calculations in the general polyhedral setting under consideration, without any 
qualification conditions, and also derive more convenient formulas in the case when the generating elements $x_{i}^{*}$ in (3.1) are linearly independent along the active constraints.

Let us start with deriving a representation of our basic/limiting normal cone (2.2) to the graph of $\mathcal{F}$ via collections of active indices at the reference point and establishing a certain stability property of this set in the sense defined in [5], which is equivalently simplified here in the framework of reflexive spaces.

Following [5], we say that a set $\Omega \subset X$ is dually norm-stable at $\bar{x} \in \Omega$ if the basic normal cone (2.2) admits the representation

$$
N(\bar{x} ; \Omega)=\left\{x^{*} \in X^{*} \mid \exists x_{k} \stackrel{\Omega}{\rightarrow} \bar{x}, \quad x_{k}^{*} \in \widehat{N}\left(x_{k} ; \Omega\right) \text { with }\left\|x_{k}^{*}-x^{*}\right\| \rightarrow 0 \text { as } k \rightarrow \infty\right\} .
$$

Comparing this property with definition (2.2) of the basic normal cone via the outer limit (1.3), we observe that (4.1) reads that the weak convergence on $X^{*}$ in (2.2) can be equivalently replaced by the norm convergence on $X^{*}$. Observing that property (4.1) obviously holds in finite dimensions, we refer the reader to [5] for verifiable conditions ensuring the dual norm-stability in infinite-dimensional spaces. Being applied to graphical sets, the dual norm-stability surely yields the coderivative normality (2.9) of set-valued mappings.

To formulate and prove the aforementioned result on computing the limiting normal cone to the graph of $\mathcal{F}$, we need the following additional constructions described entirely in terms of the initial data of (3.1). Fix an index collection $Q \subset T$, form the cone

$$
C_{Q}:=\left\{x \in X \mid\left\langle x_{i}^{*}, x\right\rangle=0 \text { for all } i \in Q,\left\langle x_{i}^{*}, x\right\rangle<0 \text { for all } i \in T \backslash Q\right\},
$$

and, given $\left(\bar{x}, \bar{x}^{*}\right) \in \operatorname{gph} \mathcal{F}$, consider the family of indices

$$
\mathcal{I}\left(\bar{x}, \bar{x}^{*}\right):=\left\{P \subset I(\bar{x}) \mid \bar{x}^{*} \in \operatorname{cone}\left\{x_{i}^{*} \mid i \in P\right\}\right\} .
$$

Theorem 4.1 (representation of basic normals to the graph and stability property for the normal cone mapping). Let $\left(\bar{x}, \bar{x}^{*}\right) \in \operatorname{gph} \mathcal{F}$ for the normal cone mapping (1.2) generated by the convex polyhedron (3.1), let $I=I(\bar{x})$ be given in (3.3), $C_{Q}$ be given in (4.2), and $\mathcal{I}=\mathcal{I}\left(\vec{x}, \bar{x}^{*}\right)$ be given in (4.3). Then the graphical set gph $\mathcal{F} \subset X \times X^{*}$ is dually norm-stable at $\left(\bar{x}, \bar{x}^{*}\right)$ and the basic normal cone to this set is represented by

$$
N\left(\left(\bar{x}, \bar{x}^{*}\right) ; \operatorname{gph} \mathcal{F}\right)=\bigcup_{P \subset Q \subset I, P \in \mathcal{I}, C_{Q} \neq \emptyset} A_{Q, P} \times B_{Q, P},
$$

where $A_{Q, P}$ and $B_{Q, P}$ are defined in (3.14) and (3.15), respectively.

Proof. In what follows we verify representation (4.4) of the basic normal cone to the graph of $\mathcal{F}$ and justify simultaneously the dual norm-stability property of the graph in question.

Let us start with proving the inclusion " $C$ " in (4.4). Pick an arbitrary limiting normal $\left(v^{*}, u\right) \in N\left(\left(\bar{x}, \bar{x}^{*}\right) ; \operatorname{gph} \mathcal{F}\right)$ and find by definition $(2.2)$ sequences $\left(x_{k}, z_{k}^{*}\right) \stackrel{\operatorname{gph} \mathcal{F}}{\longrightarrow}\left(\bar{x}, \bar{x}^{*}\right)$ and $\left(v_{k}^{*}, u_{k}\right) \stackrel{w \times w}{\longrightarrow}\left(v^{*}, u\right)$ as $k \rightarrow \infty$ satisfying

$$
\left(v_{k}^{*}, u_{k}\right) \in \widehat{N}\left(\left(x_{k}, z_{k}^{*}\right) ; \operatorname{gph} \mathcal{F}\right) \text { for all } k \in \mathbb{N} .
$$


It follows from (4.5) due to (1.2) that $x_{k} \in \Theta$ and $z_{k}^{*} \in N\left(x_{k} ; \Theta\right)$ as $k \in \mathbb{N}$. Furthermore, taking into account that there are finitely many generating elements $x_{i}^{*}$ of the convex polyhedron (3.1) and considering a subsequence of $k \in \mathbb{N}$ if necessary, assume with no loss of generality that there is a constant index subset $Q \subset I(\bar{x})$ such that

$$
Q:=\left\{i \in T \mid\left\langle x_{i}^{*}, x_{k}\right\rangle=0\right\} \text { for all } k \in \mathbb{N} .
$$

It is easy to observe that the set $C_{Q}$ from (4.2) is nonempty for the index collection $Q$ defined in (4.6). Applying representation (3.5) from Proposition 3.1 to each normal $z_{k}^{*} \in N\left(x_{k} ; \Theta\right)$ from (4.5), we get the equality

$$
z_{k}^{*}=\sum_{i \in Q} \lambda_{i k} x_{i}^{*} \text { with some } \lambda_{i k} \geq 0, \quad k \in \mathbb{N},
$$

and, extracting another subsequence by the above arguments, select without loss of generality a constant index subset $P \subset Q \subset I(\bar{x})$ such that

$$
P:=\left\{i \in Q \mid \lambda_{i k}>0\right\} \text { for all } k \in \mathbb{N} .
$$

Combining (4.7) and (4.8) allows us to verify that

$$
z_{k}^{*}=\sum_{i \in P} \lambda_{i k} x_{i}^{*} \in \operatorname{cone}\left\{x_{i}^{*} \mid i \in P\right\},
$$

which implies in turn that $\bar{x}^{*} \in$ cone $\left\{x_{i}^{*} \mid i \in P\right\}$ by the closedness of finitely generated cones. This justifies that $P \in \mathcal{I}$ for $P$ and $\mathcal{I}$ defined in (4.8) and (4.3), respectively.

Now apply the prenormal cone representation (3.19) from Theorem 3.4 to $\left(v_{k}^{*}, u_{k}\right)$ in (4.5). By the structures of the index sets in (3.19), (4.6), and (4.8) we arrive at

$$
v_{k}^{*} \in A_{Q, P} \text { and } u_{k} \in B_{Q, P} \text { for all } k \in \mathbb{N} \text {, }
$$

where $Q$ and $P$ are given in (4.6) and (4.8), respectively. Observe that the set $B_{Q, P}$ is obviously weakly closed in $X$ by construction (3.15) and that the set $A_{Q, P}$ is weakly closed in $X^{*}$ due to the polarity relationship (3.16) from Lemma 3.3 and the reflexivity of $X$. Passing finally to the limit in (4.9) as $k \rightarrow \infty$, we conclude that $\left(v^{*}, u\right) \in A_{Q, P} \times B_{Q, P}$ and thus justify the inclusion " $C$ ". in (4.4).

To prove the opposite inclusion " $\supset$ " in (4.4), fix an arbitrary element

$$
\left(v^{*}, u\right) \in \bigcup_{P \subset Q \subset I, P \in \mathcal{I}, C_{Q} \neq \emptyset} A_{Q, P} \times B_{Q, P}
$$

and find therefore some index subsets $P \subset Q \subset I(\bar{x})$ such that $P \in \mathcal{I}$ and

$$
v^{*} \in A_{Q, P} \text { and } u \in B_{Q, P} \text { with } C_{Q} \neq \emptyset
$$

where the sets $C_{Q}$ and $\mathcal{I}=\mathcal{I}\left(\bar{x}, \vec{x}^{*}\right)$ are defined in (4.2) and (4.3), respectively. Take a point $\widetilde{x} \in C_{Q}$ and construct a sequence $\left\{x_{k}\right\} \subset X$ by

$$
x_{k}:=k^{-1} \widetilde{x}+\left(1-k^{-1}\right) \bar{x} \rightarrow \bar{x} \text { as } k \rightarrow \infty .
$$


Since $\left\langle x_{i}^{*}, \widetilde{x}\right\rangle=0$ for all $i \in Q$ and $\left\langle x_{i}^{*}, \widetilde{x}\right\rangle<0$ for all $i \in T \backslash Q$ by (4.2), we have $x_{k} \in C_{Q}$ as $k \in \mathbb{N}$. This implies that $x_{k} \in \Theta$ and that the set of active constraint indices $I\left(x_{k}\right)$ at $x_{k}$ reduces to $Q$ for each $k \in \mathbb{N}$. Then representation (3.5) from Proposition 3.1 gives

$$
N\left(x_{k} ; \Theta\right)=\operatorname{cone}\left\{x_{i}^{*} \mid i \in Q\right\}, \quad k \in \mathbb{N} .
$$

Observe that the inclusion $P \in \mathcal{I}=\mathcal{I}\left(\bar{x}, \bar{x}^{*}\right)$ implies by (4.3) that

$$
\bar{x}^{*}=\sum_{i \in P} \lambda_{i} x_{i}^{*} \text { with some } \lambda_{i} \geq 0
$$

Define further a sequence $\left\{z_{k}^{*}\right\} \subset X^{*}$ by

$$
z_{k}^{*}:=\sum_{i \in P}\left(\lambda_{i}+k^{-1}\right) x_{i}^{*} \text { with }\left\|z_{k}^{*}-\bar{x}^{*}\right\| \rightarrow 0 \text { as } k \rightarrow \infty
$$

and note that $z_{k}^{*} \in N\left(x_{k} ; \Theta\right)$ for all $k \in \mathbb{N}$ due to (4.12) and $P \subset Q$. Furthermore, all the coefficients from the representation of $z_{k}^{*}$ in (4.14) are positive. Taking this into account and applying Theorem 3.4 to each $\left(x_{k}, z_{k}^{*}\right)$ with the index sets $Q$ and $P$ from (4.10), we get $\widehat{N}\left(\left(x_{k}, z_{k}^{*}\right) ; \operatorname{gph} \mathcal{F}\right)=A_{Q, P} \times B_{Q, P}$ and hence

$$
\left(v^{*}, u\right) \in \widehat{N}\left(\left(x_{k}, z_{k}^{*}\right) ; \operatorname{gph} \mathcal{F}\right) \text { for all } k \in \mathbb{N} .
$$

The latter implies, by letting $k \rightarrow \infty$ and using definition (2.2) of the basic normal cone, that $\left(v^{*}, u\right) \in N\left(\left(\bar{x}, \bar{x}^{*}\right) ; g p h \mathcal{F}\right)$, which completes the proof of representation (4.4).

To finish the proof of the theorem, it remains to show that the graphical set gph $\mathcal{F}$ is dually norm-stable at $\left(\bar{x}, \bar{x}^{*}\right)$. By definition of this property we need to check that any basic normal pair $\left(v^{*}, u\right) \in N\left(\left(\bar{x}, \bar{x}^{*}\right) ; \operatorname{gph} \mathcal{F}\right)$ can be strongly (in the norm topology of $\left.X^{*} \times X\right)$ approximated by prenormal elements to the graph of $\mathcal{F}$ at points close to $\left(\bar{x}, \bar{x}^{*}\right)$. It is actually shown in the proof of the inclusion " $\supset$ " in (4.4) that each such pair $\left(v^{*}, u\right)$ satisfies inclusion (4.15), where $x_{k} \rightarrow \bar{x}$ by (4.11) and $z_{k}^{*} \rightarrow \bar{x}^{*}$ by (4.14) as $k \rightarrow \infty$ strongly $X$ and $X^{*}$, respectively. This surely justifies the dual norm-stability of the graph of the normal cone mapping $\mathcal{F}$ and ends the the proof of the theorem.

The next result establishes a simplified representation of the basic normal cone to the graph of $\mathcal{F}$ provided that the generating elements $x_{i}^{*}$ corresponding to the active constraint indices in the convex polyhedron (3.1) are linearly independent.

Theorem 4.2 (basic normals to the graph of the normal cone mapping under linear independence of active constraints). Let $\left(\bar{x}, \bar{x}^{*}\right) \in \operatorname{gph} \mathcal{F}$ in the framework of Theorem 4.1, and let $J=J\left(\bar{x}, \bar{x}^{*}\right)$ be the index set of positive multipliers defined in (3.18). Assume that the generating elements $\left\{x_{i}^{*} \mid i \in I(\bar{x})\right\}$ of (3.1) are linearly independent. Then the basic normal cone (2.2) to the graph of $\mathcal{F}$ admits the representation

$$
N\left(\left(\bar{x}, \bar{x}^{*}\right) ; \operatorname{gph} \mathcal{F}\right)=\bigcup_{J \subset P \subset Q \subset I} A_{Q, P} \times B_{Q, P}
$$


Proof. We intend to show that the general representation (4.4) of the basic normal cone reduces to the simplified and more convenient form (4.16) under the imposed linear independence condition. Let us prove first that the latter assumption implies that

$$
C_{Q} \neq \emptyset \text { whenever } Q \subset I(\bar{x})
$$

for the set $C_{Q}$ defined in (4.2). Since $\bar{x} \in C_{I}$, we obviously have (4.17) for $Q=I(\bar{x})$. Otherwise, represent the set $C_{Q}$ as

$$
C_{Q}=\left\{x \in X \mid\left\langle x_{i}^{*}, x\right\rangle \leq 0,\left\langle-x_{i}^{*}, x\right\rangle \leq 0 \text { for } i \in I \text { and }\left\langle x_{i}^{*}, x\right\rangle<0 \text { for } i \in T \backslash Q\right\}
$$

and assume, arguing by contradiction, that $C_{Q}=\emptyset$. Then Theorem 2.2 of the alternative ensures the existence of nonnegative numbers $\alpha_{i}, \widetilde{\alpha}_{i}, \beta_{j}$ for $i \in Q$ and $j \in T \backslash Q$ such that at least one of $\beta_{j}$ is not zero and

$$
\sum_{i \in Q} \alpha_{i} x_{i}^{*}-\sum_{i \in Q} \widetilde{\alpha}_{i} x_{i}^{*}+\sum_{j \in T \backslash Q} \beta_{j} x_{j}^{*}=0 .
$$

By the inclusion $Q \subset I$ and definition (3.3) of $I=I(\bar{x})$ we get from the latter identity that

$$
\sum_{j \in T \backslash I} \beta_{j}\left\langle x_{j}^{*}, \bar{x}\right\rangle=0
$$

which implies in turn the relationships

$$
\left\langle x_{j}^{*}, \bar{x}\right\rangle<0 \text { and hence } \beta_{j}=0 \text { for all } j \in T \backslash I(\bar{x}) .
$$

This allows us to deduce from (4.18) that

$$
\sum_{i \in Q}\left(\alpha_{i}-\widetilde{\alpha}_{i}\right) x_{i}^{*}+\sum_{j \in I \backslash Q} \beta_{j} x_{i}^{*}=0
$$

where at least one of the multipliers $\beta_{j}$ is not zero. The latter contradicts the linear independence assumption made and thus justifies (4.17).

To derive next the normal cone representation (4.16) from that of (4.4) in Theorem 4.1, it is sufficient to prove the equivalence

$$
P \in \mathcal{I} \Longleftrightarrow J \subset P,
$$

where $\mathcal{I}=\mathcal{I}\left(\bar{x}, \bar{x}^{*}\right)$ is defined in (4.3). Observe right away that the implication "£" in (4.19) follows immediately from representation (3.21) and the definition of $\mathcal{I}$. To justify the opposite implication " $\Longrightarrow$ " in (4.19), take any $P \subset \mathcal{J}$ and find $\gamma_{i} \geq 0$ as $i \in P$ with

$$
\bar{x}^{*}=\sum_{i \in P} \gamma_{i} x_{i}^{*} .
$$

Recalling that $P \subset I$ by definition (4.3) and taking $\lambda_{i}$ from representation (4.13), we let

$$
\mu_{i}:=\left\{\begin{array}{cl}
\lambda_{i}, & i \in J, \\
0, & i \in I \backslash J,
\end{array} \quad \nu_{i}:=\left\{\begin{array}{cl}
\gamma_{i}, & i \in P, \\
0, & i \in I \backslash P
\end{array}\right.\right.
$$


and conclude by (3.21) and (4.20) that

$$
\bar{x}^{*}=\sum_{i \in I} \mu_{i} x_{i}^{*}=\sum_{i \in I} \nu_{i} x_{i}^{*}
$$

which implies by the linear independence assumption that $\mu_{i}=\nu_{i}$ for all $i \in I$.

Assume now that $J \not \subset P$, i.e., there is an index $i \in I$ such that $i \in J \backslash P$. It gives by (3.21) and (4.21) that

$$
0<\lambda_{i}=\mu_{i}=\nu_{i}=0
$$

for this index, which is an obvious contradiction. Thus $J \subset P$, and the conclusion of the theorem follows finally from (4.17) and (4.19).

As consequences of Theorems 4.1 and 4.2 , we obtain the following representations of the basic coderivative (2.5) involving collections of active index subsets in the general case (3.1) of convex polyhedra as well as under the linear independence condition.

Corollary 4.3 (coderivative normality and coderivative representations via collections of active index subsets). Let $\left(\bar{x}, \bar{x}^{*}\right) \in \operatorname{gph} \mathcal{F}$ in the general framework of Theorem 4.1. Then the normal cone mapping $\mathcal{F}$ is coderivatively normal at $\left(\bar{x}, \bar{x}^{*}\right)$ and the basic coderivative $(2.5)$ of $\mathcal{F}$ at $\left(\bar{x}, \bar{x}^{*}\right)$ admits the representation:

$$
\begin{aligned}
D^{*} \mathcal{F}\left(\bar{x}, \bar{x}^{*}\right)(u)=\left\{v^{*} \in X^{*} \mid\right. & \left(v^{*},-u\right) \in A_{Q, P} \times B_{Q, P} \text { for some } P \subset Q \subset I \\
& \text { with } \left.P \in \mathcal{I}\left(\bar{x}, \bar{x}^{*}\right) \text { and } C_{Q} \neq \emptyset\right\} .
\end{aligned}
$$

If in addition the generating elements $\left\{x_{i}^{*} \mid i \in I(\bar{x})\right\}$ are linearly independent, then

$$
D^{*} \mathcal{F}\left(\bar{x}, \bar{x}^{*}\right)(u)=\left\{v^{*} \in X^{*} \mid\left(v^{*},-u\right) \in A_{Q, P} \times B_{Q, P} \text { for some } J \subset P \subset Q \subset I\right\}
$$

with the index subset of positive multipliers $J=J\left(\bar{x}, \bar{x}^{*}\right)$ defined in (3.18).

Proof. Representations (4.22) and (4.23) follows from the coderivative definition (2.5) and the normal cone representation (4.4) and (4.16), respectively. The coderivative normality (2.9) of $\mathcal{F}$ at $\left(\bar{x}, \bar{x}^{*}\right)$ is an immediate consequence of the dual norm-stability of the graph of $\mathcal{F}$ at this point proved in Theorem 4.1.

Our next result, important for establishing the main theorems in this section, efficiently characterizes the coderivative domain $\operatorname{Dom} D^{*} \mathcal{F}\left(\bar{x}, \bar{x}^{*}\right)$ in the general polyhedral case (3.1), i.e., describes the subset of the coderivative argument on which the coderivative is nonempty. Given an active index collection $S \subset I(\bar{x})$, we consider the closed cone

$$
\vec{C}_{S}:=\left\{x \in X \mid\left\langle x_{i}^{*}, x\right\rangle=0 \text { for all } i \in S,\left\langle x_{i}^{*}, x\right\rangle \leq 0 \text { for all } i \in T \backslash S\right\},
$$

which is the closure of the one in (4.2), and define the feature index subset for $S$ by

$$
\Upsilon(S):=\left\{i \in I(\bar{x}) \mid\left\langle x_{i}^{*}, x\right\rangle=0 \text { for all } x \in \bar{C}_{S}\right\} .
$$


Proposition 4.4 (characterization of the coderivative domain). Let $\left(\bar{x}, \bar{x}^{*}\right) \in \operatorname{gph} \mathcal{F}$ in the framework of Theorem 4.1. Then $u \in \operatorname{Dom} D^{*} \mathcal{F}\left(\bar{x}, \bar{x}^{*}\right)$ if and only if

$$
\left\langle x_{i}^{*}, u\right\rangle=0 \text { for all } i \in J \text { and }\left\langle x_{i}^{*}, u\right\rangle \geq 0 \text { for all } i \in \Upsilon(J) \backslash J
$$

where $J=J\left(\bar{x}, \bar{x}^{*}\right)$ and $\Upsilon(J)$ are defined in (3.18) and (4.25), respectively.

Proof. Let $u \in \operatorname{Dom} D^{*} \mathcal{F}\left(\bar{x}, \bar{x}^{*}\right)$, i.e., $D^{*} \mathcal{F}\left(\bar{x}, \bar{x}^{*}\right)(u) \neq \emptyset$. Applying the coderivative definition and representation (4.4) of Theorem 4.1, find $v^{*} \in X^{*}$ and indices $P \subset Q \subset I(\bar{x})$ with $C_{Q} \neq \emptyset$ and $P \in \mathcal{I}\left(\bar{x}, \bar{x}^{*}\right)$ such that

$$
\left(v^{*},-u\right) \in A_{Q, P} \times B_{Q, P} .
$$

First we show that $J \subset Q$. Indeed, fix an element $x \in C_{Q}$ and get by definition (4.2) that

$$
\left\langle x_{i}^{*}, x\right\rangle=0 \text { for all } i \in Q \text { and }\left\langle x_{i}^{*}, x\right\rangle<0 \text { for all } i \in T \backslash Q .
$$

Since $P \in \mathcal{I}\left(\bar{x}, \bar{x}^{*}\right)$, we find by (4.3) numbers $\mu_{i} \geq 0$ such that

$$
\bar{x}^{*}=\sum_{i \in P} \mu_{i} x_{i}^{*}
$$

which implies by (4.28) that $\left\langle\bar{x}^{*}, x\right\rangle=0$ due to $P \subset Q$. On the other hand, we have from the expression of $\bar{x}^{*}$ in (3.21) that

$$
0=\left\langle\vec{x}^{*}, x\right\rangle=\sum_{i \in J} \lambda_{i}\left\langle x_{i}^{*}, x\right\rangle \text { with } \lambda_{i}>0 \text { for all } i \in J .
$$

This gives that $\left\langle x_{i}^{*}, x\right\rangle=0$ whenever $i \in J$, i.e., $J \subset Q$.

To continue proving the "only if" implication in the proposition, we get from (4.27) and construction (3.15) of the set $B_{Q, P}$ that

$$
\left\langle x_{i}^{*}, u\right\rangle=0 \text { for all } i \in P \text { and }\left\langle x_{i}^{*}, u\right\rangle \geq 0 \text { for all } i \in Q \backslash P \text {. }
$$

It follows from the inclusion $J \subset Q$ that $\left\langle x_{i}^{*}, u\right\rangle \geq 0$ for all $i \in J$. This allows us to apply to $u$ the same arguments as for $x$ above and conclude that $\left\langle x_{i}^{*}, u\right\rangle=0$ whenever $i \in J$.

Observe further that for any $x$ satisfying (4.28) we have $x \in \bar{C}_{J}$ by (4.24) due to the inclusion $J \subset Q$. Let us now show that $\Upsilon(J) \subset Q$. Indeed, otherwise we choose some index $i \in \Upsilon(J) \backslash Q$ and by definition (4.25) get $\left\langle x_{i}^{*}, x\right\rangle=0$, which clearly contradicts the inclusion $i \notin Q$. It follows then from (4.29) that $\left\langle x_{i}^{*}, u\right\rangle \geq 0$ for all $i \in \Upsilon(J) \backslash J$. Thus we arrive at (4.26) and justify the "only if" part of the proposition.

Let us prove the "if" part of the proposition assuming that the relationships in (4.26) are satisfied for the given point $u \in X$. Put $P:=J \in \mathcal{I}$ and $Q:=\Upsilon(J)$ and observe that $-u \in B_{Q, P}$ for the selected pair $(Q, P)$. Since by definition (3.14) we have $0 \in A_{Q, P}$, even for $P=\emptyset$ and/or $Q=\emptyset$ by the convention made, it follows that $(0,-u) \in A_{Q, P} \times B_{Q, P}$. By Theorem 4.1 we are done while showing that $C_{Q} \neq \emptyset$; indeed, in this case $0 \in D^{*} \mathcal{F}\left(\bar{x}, \bar{x}^{*}\right)(u)$.

To construct $x \in C_{Q}$, observe from definition (4.25) of the feature index subset that

$$
\text { for every } i \in I \backslash Q=I \backslash \Upsilon(J) \text { there is } x_{i} \in \bar{C}_{J} \text { with }\left\langle x_{i}^{*}, x_{i}\right\rangle<0 \text {. }
$$


For indices $i \in T \backslash I$ we put $x_{i}:=\bar{x} \in \bar{C}_{J}$ and thus extend the latter relationship to:

$$
\text { for every } i \in T \backslash Q \text { there is } x_{i} \in \bar{C}_{J} \text { with }\left\langle x_{i}^{*}, x_{i}\right\rangle<0 \text {. }
$$

Letting finally $x:=\sum_{i \in T \backslash Q} x_{i} \in \bar{C}_{J}$ and using (4.30) as well as definition (4.25), we get

$$
\begin{gathered}
\left\langle x_{i}^{*}, x\right\rangle=0 \text { for all } i \in Q=\Upsilon(J) \text { and } \\
\left\langle x_{i}^{*}, x\right\rangle=\left\langle x_{i}^{*}, x_{i}\right\rangle+\sum_{j \in T \backslash Q, i \neq j}\left\langle x_{i}^{*}, x_{j}\right\rangle<0 \text { for all } i \in T \backslash Q,
\end{gathered}
$$

which gives $x \in C_{Q}$ and thus completes the proof of the proposition.

Now we are ready to establish the main results of this section providing efficient evaluations of the basic coderivative $D^{*} \mathcal{F}\left(\bar{x}, \bar{x}^{*}\right)(u)$ of the normal cone mapping (1.2) entirely in terms of the initial data of the convex polyhedron (3.1), the reference point $\left(\bar{x}, \bar{x}^{*}\right) \in \operatorname{gph} \mathcal{F}$, and the coderivative argument $u \in \operatorname{Dom} D^{*} \mathcal{F}\left(\bar{x}, \bar{x}^{*}\right)$ from its domain. Given $u \in X$, define the characteristic active index subsets

$$
I_{0}(u):=\left\{i \in I(\bar{x}) \mid\left\langle x_{i}^{*}, u\right\rangle=0\right\} \text { and } I_{>}(u):=\left\{i \in I(\vec{x}) \mid\left\langle x_{i}^{*}, u\right\rangle>0\right\} .
$$

The next theorem provides a constructive upper estimate of the coderivative on its domain in the general polyhedral case (3.1) under consideration.

Theorem 4.5 (constructive upper estimate of the coderivative for the normal cone mapping with no constraint qualifications). Let $\left(\vec{x}, \bar{x}^{*}\right) \in \operatorname{gph} \mathcal{F}$ in the framework of Proposition 4.4, and let $I_{0}(u)$ and $I_{>}(u)$ be the characteristic active index subsets defined in (4.31). Then we have the coderivative upper estimate for $u \in \operatorname{Dom} D^{*} \mathcal{F}\left(\bar{x}, \bar{x}^{*}\right)$ :

$$
D^{*} \mathcal{F}\left(\bar{x}, \bar{x}^{*}\right)(u) \subset \text { cone }\left\{x_{i}^{*} \mid i \in I_{0}(u)\right\}+\operatorname{span}\left\{x_{i}^{*} \mid i \in I_{>}(u)\right\},
$$

where the coderivative domain is computed by

$$
\operatorname{Dom} D^{*} \mathcal{F}\left(\bar{x}, \bar{x}^{*}\right)=\left\{u \in X \mid\left\langle x_{i}^{*}, u\right\rangle=0, i \in J, \text { and }\left\langle x_{i}^{*}, u\right\rangle \geq 0, i \in \Upsilon(J) \backslash J\right\} \text {. }
$$

Proof. The precise domain formula (4.33) is justified in Proposition 4.4. Pick now arbitrary elements $u \in \operatorname{Dom} D^{*} \mathcal{F}\left(\bar{x}, \bar{x}^{*}\right)$ and $v^{*} \in D^{*} \mathcal{F}\left(\bar{x}, \bar{x}^{*}\right)(u)$ and find, by the coderivative definition (2.5) and description (4.4) of the basic normal cone in Theorem 4.1, such index subsets $P \subset Q \subset I(\bar{x})$ that $P \in \mathcal{I}\left(\bar{x}, \bar{x}^{*}\right), C_{Q} \neq \emptyset$,

$$
v^{*} \in A_{Q, P}, \text { and }-u \in B_{Q, P} \text {. }
$$

It follows from definition (3.15) of the set $B_{Q, P}$ that the last inclusion is equivalent to

$$
\left\langle x_{i}^{*}, u\right\rangle=0 \text { for all } i \in P \text { and }\left\langle x_{i}^{*}, u\right\rangle \geq 0 \text { for all } i \in Q \backslash P \text {. }
$$

Thus we have the following relationships involving the above vector $u \in \operatorname{Dom} D^{*} \mathcal{F}\left(\bar{x}, \bar{x}^{*}\right)$ as well as the index sets $P$ and $Q$ :

$$
P \subset S:=\left\{i \in Q \mid\left\langle x_{i}^{*}, u\right\rangle=0\right\} \text { and }\left\langle x_{i}^{*}, u\right\rangle>0 \text { for all } i \in Q \backslash S \text {. }
$$


Taking into account the relationships in (4.35) and definition (3.14) of the set $A_{Q, P}$, we derive from the first inclusion in (4.34) that

$$
\begin{aligned}
v^{*} & \in \operatorname{span}\left\{x_{i}^{*} \mid i \in P\right\}+\operatorname{cone}\left\{x_{i}^{*} \mid i \in Q \backslash P\right\} \\
& \subset \operatorname{span}\left\{x_{i}^{*} \mid i \in S\right\}+\operatorname{cone}\left\{x_{i}^{*} \mid i \in Q \backslash S\right\} .
\end{aligned}
$$

Observe further from the constructions of $S$ in (4.35) and of the characteristic active index subsets in (4.31) that $S \subset I_{0}(u)$ and $Q \backslash S \subset I_{>}(u)$. Thus we get (4.32) from (4.36) and complete the proof of the theorem.

The final result of this section establishes a precise formula for computing the coderivative of the normal cone mapping $\mathcal{F}$ at $\left(\bar{x}, \bar{x}^{*}\right)$ provided that the generating elements $x_{i}^{*}$ of the convex polyhedron (3.1) are linearly independent along the active constraints at $\bar{x}$.

Theorem 4.6 (computing the coderivative of the normal cone mapping under linear independence of active constraints). Assume in the framework of Theorem 4.5 that the generating element $\left\{x_{i}^{*} \mid i \in I(\bar{x})\right\}$ of (3.1) are linearly independent. Then we have

$$
D^{*} \mathcal{F}\left(\bar{x}, \bar{x}^{*}\right)(u)=\text { cone }\left\{x_{i}^{*} \mid i \in I_{0}(u)\right\}+\operatorname{span}\left\{x_{i}^{*} \mid i \in I_{>}(u)\right\}
$$

for all $u \in \operatorname{Dom} D^{*} \mathcal{F}\left(\bar{x}, \bar{x}^{*}\right)$, where the coderivative domain is computed in (4.33).

Proof. By Theorem 4.5 it remains to justify the opposite inclusion " $\supset$ " to (4.32) under the imposed linear independence condition. It easily follows from the definitions that $\Upsilon(J)=J$ for the feature index subset (4.25) of $J=J\left(\bar{x}, \bar{x}^{*}\right)$ in (3.18) under the assumed linear independence of the generating elements $\left\{x_{i}^{*} \mid i \in I(\bar{x})\right\}$. Take $\left(v^{*}, u\right)$ satisfying.

$$
v^{*} \in \text { cone }\left\{x_{i}^{*} \mid i \in I_{0}(u)\right\}+\operatorname{span}\left\{x_{i}^{*} \mid i \in I_{>}(u)\right\}
$$

and observe by (3.14) and (3.15) that the latter inclusion yields

$$
\left(v^{*},-u\right) \in A_{Q, P} \times B_{Q, P} \text { with } Q:=I_{0}(u) \cup I_{>}(u) \text { and } P:=I_{0}(u)
$$

via the characteristic active index subsets (4.31). Since

$$
J \subset I_{0}(u) \subset I_{0}(u) \cup I_{>}(u) \subset I,
$$

we derive the inclusion " $\supset$ " in (4.37) from the relationships in (4.38) and the coderivative representation (4.22) of Corollary 4.3 and thus complete the proof of the theorem.

Let us conclude this section with three extended remarks, which compare the results obtained with known in the literature, relate the main theorems to the second-order subdifferentials mentioned in Introduction, and discuss some applications.

Remark 4.7 (comparison with known results). As mentioned in Section 1, all the results obtained in both Section 3 and Section 4 are new in infinite dimensions. In this paper the results of Section 3, which are of their own interest, play an auxiliary role as a necessary preliminary step for computing, according to the definitions, the basic normal 
cone and coderivative in infinite dimensions. In finite-dimensional spaces there are analogs and versions of some results obtained above discussed in what follows.

Proposition 3.2 is implicitly given in [3] in finite dimensions and then explicitly proved by a different way in [27] in the same setting. Our proof in reflexive spaces mainly follows the approach of [27]. The other results of Section 3 seem to be new even in finite dimensions.

The first representation of the normal cone (2.2) to the graph of $\mathcal{F}=N(x ; \Theta)$ is given in [3, proof of Theorem 2] via some closed face description of convex polyhedra, which is generally difficult to check. However, it is shown in [7, Proposition 3.2] that the closed face representation of [3] is equivalent to an explicit one, which is of the same type but somewhat different from the finite-dimensional analog of our Theorem 4.4. Another proof of a similar while not fully explicit normal cone representation in $\mathbb{R}^{n}$ is independently derived in $[27$, Theorem 3.3]. Note that the proof in [27] as well as our proof in infinite dimensions do not use the rather involved Reduction Lemma and other devices from [3].

The coderivative representation of Theorem 4.6 under the linear independence condition is an infinite-dimensional extension of that in [7, Corollary 3.5]. The other results of Section 4 seem to be new in finite dimensions while Theorem 3.5 is an improved version of [7, Corollary 3.4]. Note also that the recent paper [6] establishes efficient coderivative descriptions of the normal cone mapping for nonpolyhedral inequality systems described by smooth nonlinear functions in finite dimensions under certain qualification conditions. These new developments are largely based on the methods and results from $[7,17,18]$.

Remark 4.8 (second-order subdifferentials). Given an extended-real-valued function $\varphi: X \rightarrow \overline{\mathbb{R}}:=(-\infty, \infty]$ finite at $\bar{x} \in \overline{\mathbb{R}}$, we recall the notions if the (first-order) subdifferential [11] and the second-order subdifferential [13] of $\varphi$ generated by the basic normal cone (2.2); the reader can find equivalent representations, more details and discussions, various calculus rules, and numerous applications in $[15,16]$ in the references therein. The basic subdifferential of $\varphi$ at $\bar{x}$ is defined by

$$
\partial \varphi(\bar{x}):=\left\{x^{*} \in X^{*} \mid\left(x^{*},-1\right) \in N((\bar{x}, \varphi(\bar{x})) ; \text { epi } \varphi)\right\}
$$

via the normal cone to the epigraphical set epi $\varphi:=\{(x, \mu) \in X \times \mathbb{R} \mid \mu \geq \varphi(x)\}$. It is easy to see the subdifferential representation of the normal cone

$$
N(\bar{x} ; \Omega)=\partial \delta(\bar{x} ; \Omega), \quad \bar{x} \in \Omega,
$$

where $\delta(\cdot ; \Omega)$ is the indicator function of the set $\Omega$ equal 0 for $x \in \Omega$ and $\infty$ otherwise. Given further $\left(\bar{x}, \bar{x}^{*}\right) \in$ gph $\partial \varphi$, define the second-order subdifferential of $\varphi$ at this point as the coderivative (2.5) of the first-order subdifferential mapping $\partial \varphi: X \rightrightarrows X^{*}$ at $\left(\bar{x}, \bar{x}^{*}\right)$ by

$$
\partial^{2} \varphi\left(\bar{x}, \bar{x}^{*}\right)(u):=\left(D^{*} \partial \varphi\right)\left(\bar{x}, \bar{x}^{*}\right)(u), \quad u \in X\left(=X^{* *}\right) .
$$

Construction (4.41) accumulating second-order information on the function in question is a natural development of the classical "derivative-of-derivative" approach to (generalized) second-order differentiation; see $[13,15,18,21,25]$ for more discussions and implementations. It follows from (4.40) and (4.41) that the coderivative of the normal cone mapping $N(x ; \Omega)$ to a set $\Omega$ can be interpreted as the the second-order subdifferential of the indicator 
function of $\Omega$ at the corresponding point. Note that such second-order constructions naturally appear in optimization and sensitivity analysis of parametric variational inequalities and related problems known as mathematical and equilibrium programs with equilibrium constraints (MPECs and EPECs); see, e.g., $[4,15,16,19,29]$ and the references therein.

From this viewpoint, the results obtained in Section 4 as well as their finite-dimensional predecessors from $[3,6,7,27,28]$ can be treated as constructive tools for efficient computing the second-order subdifferentials of the indicator functions for convex polyhedra. We thus make the first attempt for such a constructive second-order analysis in infinite dimensions.

Remark 4.9 (some applications). The primary motivation for this paper is developing applications to robust stability of parametric variational inequalities, which are presented in the next section. At the same time the constructive coderivative calculations of Section 4 can be readily applied to other important issues of variational analysis and optimization. In particular, based on these calculations and the general approaches and results developed in [16, Chapter 5], we can derive constructive necessary optimality conditions for MPECs and EPECs with equilibrium constraints governed by parametric generalized equations

$$
0 \in f(x, p)+N(x ; \Theta) \text {, }
$$

where $\Theta$ is the convex polyhedral (3.1) in a reflexive Banach space. Recall that Robinson's generalized equation model (4.42) encompasses variational inequalities over polyhedral convex sets and has been well recognized as a convenient framework for the study of both qualitative and numerical aspects of variational analysis, optimization, and equilibria; see, e.g., $[4,16,19,23]$ and the references therein.

Furthermore, following the scheme developed in [7] for finite-dimensional models, the results obtained above have the potential for applications to deriving constructive optimality and stationarity conditions as well as their practical implementations in infinitedimensional MPECs and EPECs arising in electricity spot market modeling with timedependent/dynamic data such as demands on the network nodes, electricity generation and distribution along the arcs, etc. This will be considered in detail in our future research.

\section{Robust Stability of Parametric Variational Inequalities}

The concluding section of the paper is devoted to applications of the coderivative calculations in Section 4 to constructive characterizing robust stability-via the general criteria of Theorem 2.1-of parametric variational inequalities given in the generalized equation form:

$$
0 \in f(p, x)+N(x ; \Theta) \text { for } x \in \Theta \text { and } p \in Z \text {, }
$$

where $\Theta \subset X$ is the convex polyhedron (3.1), and where $f: Z \times X \rightarrow X^{*}$ is a continuous (with respect to the norm topologies) mapping depending on the decision variable $x$ and the parameter variable $p$ taking values in the corresponding reflexive Banach spaces. Note that, by construction (3.2) of the normal cone of convex analysis, the generalized equation form (5.1) is equivalent to the standard form of variational inequalities over convex sets:

$$
\langle f(p, x), x-u\rangle \leq 0 \text { for all } u \in \Theta
$$


with $x \in \Theta$ and $p \in Z$. Define further the parametric solution map $S: Z \rightrightarrows X$ to (5.1) by

$$
S(p):=\{x \in X \mid 0 \in f(p, x)+N(x ; \Theta)\},
$$

where we have in fact $x \in \Theta$, since $N(x ; \Theta)=\emptyset$ for $x \notin \Theta$.

Our primary goal in what follows is to derive constructive characterizations of the Lipschitz-like property of the solution map (5.3) with evaluating the exact Lipschitzian bound in (2.11) entirely in terms of the initial data of (5.1) in both finite and infinite dimensions. This will be done by combining the criteria of Theorem 2.1, some calculus results from [15], and the coderivative calculations of Section 4.

Let us first check that the general assumptions of Theorem 2.1 are satisfied for the solution map $S: Z \rightrightarrows X$ from (5.3).

Lemma 5.1 (closed graph and coderivative normality properties of solution maps). The graph $\operatorname{gph} S \subset Z \times X$ of the solution map (5.3) is always closed in $Z \times X$. Furthermore, the mapping $S: Z \rightrightarrows X$ is coderivatively normal at every point $(\bar{p}, \bar{x}) \in \operatorname{gph} S$ where $f$ is strictly differentiable and its partial derivative $\nabla_{p} f(\bar{p}, \bar{x}): Z \rightarrow X^{*}$ is surjective.

Proof. To prove the closedness of the graph of $S$, we get by (5.2) that

$$
\operatorname{gph} S=\{(p, x) \in Z \times \Theta \mid\langle f(p, x), x-u\rangle \leq 0 \text { for all } u \in \Theta\} .
$$

This readily implies that gph $S$ is closed due to the continuity of the base mapping $f$.

Let us next justify the coderivative normality property of $S$ under the additional assumptions on $f$ imposed at the given point $(\bar{p}, \bar{x}) \in \operatorname{gph} S$. To proceed, consider a mapping $g: Z \times X \rightarrow X \times X^{*}$ defined by

$$
g(p, x):=(x,-f(p, x)) \text { for } p \in Z \text { and } x \in X
$$

and observe that the graph of $S$ admits the representation

$$
\operatorname{gph} S=\{(p, x) \in Z \times \Theta \mid g(p, x) \in \operatorname{gph} \mathcal{F}\}=g^{-1}(\operatorname{gph} \mathcal{F})
$$

via the inverse image/preimage of the graph of the normal cone mapping $\mathcal{F}(x)=N(x ; \Theta)$ under the mapping $g$ from (5.4). It is easy to see that $g$ is strictly differentiable at $(\bar{p}, \bar{x})$ due to the this property of $f$ and that the (full) derivative $\nabla g(\bar{p}, \bar{x}): Z \times X \rightarrow X \times X^{*}$ of $g$ at $(\bar{p}, \bar{x})$ is surjective by the surjectivity assumption imposed on the partial derivative of $\nabla_{p} f(\bar{p}, \bar{x})$. Employing the inverse image rule for basic normals from [15, Theorem 1.17] to the inverse image representation in (5.5), we get the equality

$$
N((\bar{p}, \bar{x}) ; \operatorname{gph} S)=\nabla g(\bar{p}, \bar{x})^{*} N((\bar{x},-f(\bar{p}, \bar{x})) ; \operatorname{gph} \mathcal{F})
$$

Based on representation (5.6) and the surjectivity of $\nabla g(\bar{p}, \bar{x})$, let us now prove that the graph of the solution map $S$ enjoys the dual norm-stability property $(4.1)$ at $(\bar{p}, \bar{x})$, which obviously implies the coderivative normality of $S$ at the reference point. Take $\left(p^{*}, x^{*}\right) \in N((\bar{p}, \bar{x}) ; \operatorname{gph} S)$. By $(5.6)$ there is a pair $\left(u^{*}, v^{*}\right) \in N((\bar{x},-f(\bar{p}, \bar{x})) ; \operatorname{gph} \mathcal{F})$ 
such that $\left(p^{*}, x^{*}\right)=\nabla g(\bar{p}, \bar{x})^{*}\left(u^{*}, v^{*}\right)$. Since $\nabla g(\bar{p}, \bar{x})$ is surjective, the pair $\left(u^{*}, v^{*}\right)$ is determined uniquely; see [15, Lemma 1.18]. As proved in Theorem 4.1, the set gph $\mathcal{F}$ is dually norm-stable at $(\bar{x},-f(\bar{p}, \bar{x}))$. Thus there are sequences $\left(u_{k}, v_{k}\right) \rightarrow(\bar{x},-f(\bar{p}, \bar{x}))$ with $\left(u_{k}, v_{k}\right) \in \operatorname{gph} \mathcal{F}$ and $\left\{\left(u_{k}^{*}, v_{k}^{*}\right)\right\} \subset X^{*} \times X$ such that

$$
\left(u_{k}^{*}, v_{k}^{*}\right) \in \hat{N}\left(\left(u_{k}, v_{k}\right) ; \operatorname{gph} \mathcal{F}\right) \text { and }\left\|\left(u_{k}^{*}, v_{k}^{*}\right)-\left(u^{*}, v^{*}\right)\right\| \rightarrow 0 \text { as } k \rightarrow \infty
$$

Define further $\left(p_{k}^{*}, x_{k}^{*}\right):=\nabla g(\bar{p}, \bar{x})^{*}\left(u_{k}^{*}, v_{k}^{*}\right)$ for all $k \in \mathbb{N}$ and observe by (5.7) that

$$
\left(p_{k}^{*}, x_{k}^{*}\right) \in \nabla g(\bar{p}, \bar{x})^{*} \widehat{N}\left(\left(u_{k}, v_{k}\right) ; \operatorname{gph} \mathcal{F}\right) \text { and }\left\|\left(p_{k}^{*}, x_{k}^{*}\right)-\left(p^{*}, x^{*}\right)\right\| \rightarrow 0 \text { as } k \rightarrow \infty \text {. }
$$

It follows from (5.8) by [15, Lemma 1.16] that there are $\left(\widetilde{u}_{k}, \widetilde{v}_{k}\right) \rightarrow(\vec{x},-f(\bar{p}, \bar{x}))$ with $\left(\widetilde{u}_{k}, \widetilde{v}_{k}\right) \in \operatorname{gph} \mathcal{F}$ and

$$
\left(\widetilde{p}_{k}^{*}, \widetilde{x}_{k}^{*}\right) \in \widehat{N}\left(\left(\widetilde{p}_{k}, \widetilde{v}_{k}\right) ; \operatorname{gph} S\right) \text { such that }\left\|\left(\widetilde{p}_{k}^{*}, \widetilde{x}_{k}^{*}\right)-\left(p^{*}, x^{*}\right)\right\| \rightarrow 0 \text { as } k \rightarrow \infty .
$$

This justifies the dual norm-stability property of the graph of the solution map $S$ at $(\bar{p}, \bar{x})$ and thus completes the proof of the lemma.

Our next results presented in the following proposition provide constructive representations of the basic coderivative (2.5) of the solution map (5.3) via the initial data of the variational inequality (5.1) under consideration. Based on the coderivative representations for the normal cone mapping $\mathcal{F}(x)=N(x ; \Theta)$ from Section 4, we consider the two cases: the general polyhedra (3.1) without any qualification conditions and the case of linearly independent generating elements $x_{i}^{*}$ corresponding to active constraints. In the first case we involve collections of active index subsets, while the second one allows us to derive a precise coderivative representation using only characteristic active index subsets defined in (4.31). The results obtained, being of their own interest, are motivated here by applications to robust stability to variational inequalities via the criteria of Theorem 2.1 .

Proposition 5.2 (computing the coderivative of solution maps to variational inequalities). Let $(\bar{p}, \bar{x}) \in \operatorname{gph} S$ for the solution map (5.3), where $f$ is strictly differentiable at $(\bar{p}, \bar{x})$ with the surjective partial derivative $\nabla_{p} f(\bar{p}, \bar{x})$. Let $\bar{x}^{*}:=-f(\bar{p}, \bar{x})$ in the notation of Corollary 4.3. Then the following assertions hold:

(i) The coderivative $D^{*} S(\bar{p}, \bar{x}): X^{*} \rightrightarrows Z^{*}$ is computed by

$D^{*} S(\bar{p}, \bar{x})\left(x^{*}\right)=\left\{\begin{array}{l}p^{*} \in Z^{*} \mid \exists u \in X, P \subset Q \subset I \text { with } P \in \mathcal{I}, C_{Q} \neq \emptyset \\ \text { s.t. }\left(-x^{*}-\nabla_{x} f(\bar{p}, \bar{x})^{*} u,-u\right) \in A_{Q, P} \times B_{Q, P}, p^{*}=\nabla_{p} f(\bar{p}, \bar{x})^{*} u .\end{array}\right.$

(ii) Assume in addition that the generating element $\left\{x_{i}^{*} \mid i \in I(\bar{x})\right\}$ of the convex polyhedron (3.1) are linearly independent. Then the coderivative $D^{*} S(\bar{p}, \bar{x})$ is computed by

$$
D^{*} S(\bar{p}, \bar{x})\left(x^{*}\right)=\left\{\begin{array}{l}
p^{*} \in Z^{*} \mid \exists u \in \operatorname{Dom} D^{*} \mathcal{F}\left(\bar{x}, \bar{x}^{*}\right) \text { with } p^{*}=\nabla_{p} f(\bar{p}, \bar{x})^{*} u \text { and } \\
\left.-x^{*}-\nabla_{x} f(\bar{p}, \bar{x})^{*} u \in \operatorname{cone}\left\{x_{i}^{*} \mid i \in I_{0}(u)\right\}+\operatorname{span} x_{i}^{*} \mid i \in I_{>}(u)\right\}
\end{array}\right.
$$

where the characteristic active index subsets $I_{0}(u)$ and $I_{>}(u)$ are defined in (4.31) while the coderivative domain $\operatorname{Dom} D^{*} \mathcal{F}\left(\bar{x}, \bar{x}^{*}\right)$ is computed in (4.33). 
Proof. It follows from [15, Theorem 4.44] that, under the strict differentiability and surjectivity assumptions made in this proposition, we have the coderivative representation of the solution map $S$ to the variational inequality/generalized equation (5.1):

$$
D^{*} S(\bar{p}, \bar{x})\left(x^{*}\right)= \begin{cases}p^{*} \in Z^{*} \mid & \exists u \in X \text { with } p^{*}=\nabla_{p} f(\bar{p}, \bar{x})^{*} u, \\ & -x^{*}-\nabla_{x} f(\bar{p}, \bar{x})^{*} u \in D^{*} \mathcal{F}\left(\bar{x}, \bar{x}^{*}\right)(u) .\end{cases}
$$

Then we arrive at both coderivative formulas in (i) and (ii) of the proposition by substituting into (5.9) the representations of $D^{*} \mathcal{F}\left(\bar{x}, \bar{x}^{*}\right)$ from (4.22) of Corollary 4.3 and from (4.37) of Theorem 4.6, respectively. This completes the proof of the proposition.

Now we are ready to establish verifiable characterizations for robust Lipschitzian stability of solution maps to the variational inequalities (5.1) over polyhedral convex sets with evaluating the exact Lipschitzian bound. Let us first consider the case when the decision space $X$ is finite-dimensional while the parameter space $Z$ may be arbitrary Banach and reflexive. We include two statements into the next theorem: one for the general polyhedral set (3.1) with no qualification conditions and the other under the linear independence of generating elements of the convex polyhedron (3.1).

Theorem 5.3 (constructive characterizations of robust stability of polyhedral variational inequalities with finite-dimensional decision spaces). Take the reference point $(\bar{p}, \bar{x}) \in \operatorname{gph} S$ in the framework and notation of Proposition 5.2 and assume that the decision space $X$ is finite-dimensional. Then the following assertions hold:

(i) The solution map (5.3) is Lipschitz-like around $(\bar{p}, \bar{x})$ if and only if

$$
\left[-\nabla_{x} f(\bar{p}, \bar{x})^{*} u \in A_{Q, P}, \quad-u \in B_{Q, P}\right] \Longrightarrow u=0
$$

for all $P \subset Q \subset I(\bar{x})$ with $P \in \mathcal{I}\left(\bar{x}, \bar{x}^{*}\right)$ and $C_{Q} \neq \emptyset$. Furthermore, we have the lower estimate of the exact Lipschitzian bound for $S$ at $(\bar{p}, \bar{x})$ :

$$
\begin{aligned}
& \operatorname{lip} S(\bar{p}, \bar{x}) \geq \max \left\{\left\|\nabla_{p} f(\bar{p}, \bar{x})^{*} u\right\| \mid u \in-B_{Q, P}, x^{*} \in-\nabla_{x} f(\bar{p}, \bar{x})^{*} u-A_{Q, P},\right. \\
& \left\|\nabla_{x} f(\bar{p}, \bar{x})^{*} u+x^{*}\right\| \leq 1, P \subset Q \subset I(\bar{x}) \\
& \text { with } \left.P \in \mathcal{I}\left(\bar{x}, \bar{x}^{*}\right) \text { and } C_{Q} \neq \emptyset\right\} \text {, }
\end{aligned}
$$

which holds as equality if the parameter space $Z$ is finite-dimensional.

(ii) Assume in addition that the generating elements $\left\{x_{i}^{*} \mid i \in I(\bar{x})\right\}$ of (3.1) are linearly independent. Then $S$ is Lipschitz-like around $(\bar{p}, \bar{x})$ if and only if

$$
\left[-\nabla_{x} f(\bar{p}, \bar{x})^{*} u \in \operatorname{cone}\left\{x_{i}^{*} \mid i \in I_{0}(u)\right\}+\operatorname{span}\left\{x_{i}^{*} \mid i \in I_{>}(u)\right\}\right] \Longrightarrow u=0
$$

provided that $u \in \operatorname{Dom} \mathcal{F}\left(\bar{x}, \bar{x}^{*}\right)$, where the characteristic index subsets $I_{0}(u)$ and $I_{>}(u)$ are defined in (4.31) while the coderivative domain $\operatorname{Dom} D^{*} \mathcal{F}\left(\bar{x}, \bar{x}^{*}\right)$ is computed in (4.33). In fact, implication (5.12) with $u \in \operatorname{Dom} D^{*} \mathcal{F}\left(\bar{x}, \bar{x}^{*}\right)$ is equivalent to

$$
\left[-\nabla_{x} f(\bar{p}, \bar{x})^{*} u \in A_{I, I}, \quad-u \in B_{J, J}\right] \Longrightarrow u=0
$$

with $I=I(\bar{x})$ and $J=J\left(\bar{x}, \bar{x}^{*}\right)$ as well as to the condition

$$
\operatorname{ker}\left\{x_{i}^{*} \mid i \in J\left(\bar{x}, \bar{x}^{*}\right)\right\} \bigcap\left[\nabla_{x} f(\bar{p}, \bar{x})^{*}\right]^{-1}\left(\operatorname{span}\left\{x_{i}^{*} \mid i \in I(\bar{x})\right\}\right)=\{0\}
$$


involving the inverse operator to $\nabla_{x} f(\bar{p}, \bar{x})^{*}$. Furthermore, we have the lower estimate

$$
\begin{aligned}
\operatorname{lip} S(\bar{p}, \bar{x}) \geq \max \{ & \left\|\nabla_{p} f(\bar{p}, \bar{x})^{*} u\right\| \mid u \in \operatorname{Dom} D^{*} \mathcal{F}\left(\bar{x}, \bar{x}^{*},\left\|\nabla_{x} f(\bar{p}, \bar{x})^{*} u+x^{*}\right\| \leq 1,\right. \\
& \left.-x^{*}-\nabla_{x} f(\bar{p}, \bar{x})^{*} u \in \operatorname{cone}\left\{x_{i}^{*} \mid i \in I_{0}(u)\right\}+\operatorname{span}\left\{x_{i}^{*} \mid i \in I_{>}(u)\right\}\right\}
\end{aligned}
$$

for the exact Lipschitzian bound of $S$ at $(\bar{p}, \bar{x})$, which holds as equality when the parameter space $Z$ is finite-dimensional.

Proof. Observe first that the general assumptions of Theorem 2.1 are satisfied by Lemma 5.1. Note also that the PSNC property of $S$ is automatic when the decision/range space $X$ is finite-dimensional and that the condition $\nabla_{p} f(\bar{p}, \bar{x})^{*} u=0$ is equivalent to $u=0$ due to the surjectivity of $\nabla_{p} f(\bar{p}, \bar{x})$. Thus the necessary and sufficient conditions (5.10) and (5.12) for the Lipschitz-like property of $S$ in (i) and (ii), respectively, follow directly from the coderivative criterion (2.12) of Theorem 2.1 and the coderivative formulas for $S$ derived in Proposition 5.2 as $x^{*}=0$. Further, it follows from the proof of Theorem 4.6 and the obvious set monotonicity relationships

$$
A_{L, M} \subset A_{L^{\prime}, M^{\prime}} \text { and } B_{L, M} \supset B_{L^{\prime}, M^{\prime}} \text { whenever } L \subset L^{\prime}, M \subset M^{\prime}
$$

for the constructions in (3.14) and (3.15) that the robust stability criterion (5.12) can be equivalently written in the form of (5.13). The equivalence between conditions (5.13) and (5.14) directly follows from definitions (3.14) and (3.15). Using finally the coderivative formulas from Proposition 5.2, we compute the coderivative norm by the maximum expressions in (5.11) and (5.15) under the assumptions imposed. Note that the maximum is realized in these formulas for the coderivative norm (2.13) due to [15, Theorem 4.56] and the graphclosedness of the normal cone mapping $\mathcal{F}$ in the norm $\times$ weak topology on $X \times X^{*}$, which is proved by the stability arguments in Theorem 4.1. Thus the exact bound estimates (5.11), (5.15) and the equalities therein follow from the corresponding assertions of Theorem 2.1. This completes the proof of this theorem.

Let us present a simple consequence of Theorem 5.3 ensuring the Lipschitz-like property of the parametric solution map (5.3) when all the generating elements of the convex polyhedron (3.1) are active and linearly independent and when the so-called strict complementarity condition $I(\bar{x})=J\left(\bar{x}, \bar{x}^{*}\right)$ is satisfied.

Corollary 5.4 (robust stability under strict complementarity). Assume in the framework of Theorem 5.3(ii) that $X=\mathbb{R}^{n}$ and $I(\bar{x})=J\left(\bar{x}, \bar{x}^{*}\right)=\{1, \ldots, n\}$, where $\bar{x}^{*}=-f(\bar{p}, \bar{x})$. Then the solution map $S$ to (5.1) is Lipschitz-like around $(\bar{p}, \bar{x})$.

Proof. It immediately follows from (4.33) that $\operatorname{Dom} D^{*} \mathcal{F}\left(\bar{x}, \bar{x}^{*}\right)=\{0\}$ in this case, i.e., the stability criterion (5.12) of Theorem 5.3(ii) is satisfied automatically.

Remark 5.5 (specifications and implementations of the constructive characterizations of robust stability). Based on the constructive characterizations of robust stability obtained in both assertions of Theorem 5.3 in the case of finite-dimensional decision spaces, we can derive their various specifications and simplifications in particular 
settings; Corollary 5.4 provides just a simple example of this. Observe that criterion (5.14) in Theorem 5.3(ii) can be equivalently rewritten as

$$
\left[A^{*} u-C_{2}^{*} v=0, C_{1} u=0\right] \Longrightarrow u=0,
$$

where $A:=\nabla_{x} f(\bar{p}, \bar{x})$ and where the matrices $C_{1}$ and $C_{2}$ are composed from the row vectors of the generating vector $x_{i}^{*}$ for $i \in J\left(\bar{x}, \bar{x}^{*}\right)$ and $i \in I(\bar{x})$, respectively. Assuming in addition to the linear independence of $\left\{x_{i}^{*} \mid i \in I(\bar{x})\right\}$ the strict complementarity condition $J\left(\bar{x}, \bar{x}^{*}\right)=I(\bar{x})$, we have $C_{1}=C_{2}:=C$ and get (5.17) from the positive definiteness of $A$ on the kernel subspace $\operatorname{ker}\left\{x_{i}^{*} \mid i \in I(\bar{x})\right\}$. The latter readily reduces to the classical second-order sufficient condition for local optimality in nonlinear programs written in the variational equality form (5.1) with $f$ being the gradient of an objective function; see [24]. By some more elaboration we can show that condition (5.17) is actually equivalent in the latter setting to the so-called strong second-order sufficient condition for local optimality in $C^{2}$ nonlinear programs; cf. $[3,9,24]$ with the references therein and also further discussions in Remark 5.10 below.

Next we describe general settings in which the conditions of Theorem 5.3(ii) provide characterizations of robust stability for solution maps (5.3) to the polyhedral variational inequalities (5.1) in the case of infinite-dimensional decision spaces. They rely on a certain well-posedness of (5.1) concerning behavior of the partial derivative $\nabla_{x} f(\bar{p}, \bar{x})$ of the base mapping $f$ on the kernel space formed by generating elements $x_{i}^{*}$ of the convex polyhedron (3.1) along the index subset (3.18) of positive multipliers at the reference point. This wellposedness is automatic in finite dimensions while holding under easily verifiable conditions in the case of infinite-dimensional decision spaces.

Definition 5.6 (kernel well-posedness of polyhedral variational inequalities). We say that the parametric variational inequality (5.1) over the convex polyhedron (3.1) exhibits the KERNEL WELL-POSEDNESS at the point $(\bar{p}, \bar{x}) \in \operatorname{gph} S$ of differentiability of the base mapping $f$ with respect to the decision variable if

$$
\left[\left\|\nabla_{x} f(\bar{p}, \bar{x})^{*} x_{k}\right\| \rightarrow 0, \quad x_{k} \stackrel{w}{\rightarrow} 0, \quad x_{k} \in \operatorname{ker}\left\{x_{i}^{*} \mid i \in J\left(\bar{x}, \bar{x}^{*}\right)\right\}\right] \Longrightarrow\left\|x_{k}\right\| \rightarrow 0
$$

as $k \rightarrow \infty$, where $J=J\left(\bar{x}, \bar{x}^{*}\right)$ is defined in (3.18) with $\bar{x}^{*}=-f(\bar{p}, \bar{x})$.

Observe that the introduced well-posedness property of (5.1) does not actually depend on the parameter space $Z$. Let us now present some verifiable conditions ensuring the kernel well-posedness of the polyhedral variational inequalities under consideration.

Given a linear bounded operator $A: X \rightarrow X^{*}$ on a Banach space $X$ and a closed subspace $L \subset X$, we say that $A$ is coercive on the subspace $L$ if there is a constant $\mu>0$ such that

$$
\mu\|x\|^{2} \leq\langle A x, x\rangle \text { for all } x \in L
$$

This reduces to the conventional coercivity of $A: X \rightarrow X^{*}$ when $L=X$. We use both versions in what follows; see Theorem 5.8 and Corollary 5.9. 
Proposition 5.7 (sufficient conditions for kernel well-posedness). Each of the following conditions ensures the kernel well-posedness of the polyhedral variational inequality (5.1) at $(\bar{p}, \bar{x}) \in \operatorname{gph} S$ :

(a) The decision space $X$ is finite-dimensional.

(b) The adjoint operator $\nabla_{x} f(\bar{p}, \bar{x})^{*}: X \rightarrow X^{*}$ is injective on the kernel subspace

$$
L:=\operatorname{ker}\left\{x_{i}^{*} \mid i \in J\left(\bar{x}, \bar{x}^{*}\right)\right\} \subset X
$$

of the Banach space $X$, i.e., we have

$$
\left[\nabla_{x} f(\bar{p}, \bar{x})^{*}\left(x_{1}-x_{2}\right)=0\right] \Longrightarrow\left[x_{1}=x_{2}\right] \text { for any } x_{1}, x_{2} \in L
$$

and furthermore the image subspace $\nabla_{x} f(\bar{p}, \bar{x})^{*} L$ is closed in $X^{*}$; both these properties are automatic with $L$ replaced by $X$ when the partial derivative operator $\nabla_{x} f(\bar{p}, \bar{x})$ is surjective.

(c) The operator $\nabla_{x} f(\bar{p}, \bar{x}): X \rightarrow X^{*}$ is coercive on the Banach space $X$.

Proof. Case (a) is obvious. To justify case (b), it is sufficient to show that

$$
\left[\left\|\nabla_{x} f(\bar{p}, \bar{x})^{*} x_{k}\right\| \rightarrow 0, x_{k} \in L\right] \Longrightarrow\left\|x_{k}\right\| \rightarrow 0 \text { as } k \rightarrow \infty
$$

under the injectivity and closedness assumptions made in (b). Denote $\Lambda:=\nabla_{x} f(\bar{p}, \bar{x})^{*}$ and prove that there is $\kappa>0$ such that

$$
\|\Lambda x\| \geq \kappa\|x\| \text { for all } x \in L,
$$

which surely yields (5.20). To proceed, denote $Y:=\Lambda L \subset X^{*}$ and consider the operator $A: L \rightarrow Y$. Our assumptions ensure that the set $Y$ is closed and the operator $A: L \rightarrow Y$ is invertible. By the classical open mapping theorem we conclude that the inverse operator $A^{-1}: Y \rightarrow L$ is continuous. Thus there is a constant $\nu>0$ such that $\left\|A^{-1} y\right\| \leq \nu\|y\|$ for all $y \in Y$. This implies (5.21). If $\nabla_{x} f(\bar{p}, \bar{x})$ is surjective, we have (5.21) and (5.20) with $L$ replaced by $X$ from [15, Lemma 1.18$]$.

Finally, the kernel well-posedness in case (c) follows directly from the Banach space version [22] of the classical Lax-Milgram theorem ensuring that coercivity implies surjectivity. This completes the proof of the proposition.

Now we are ready to establish constructive characterizations of robust stability for (5.1) in the general case of reflexive decision spaces.

Theorem 5.8 (constructive conditions for robust stability of well-posed polyhedral variational inequalities with infinite-dimensional decision spaces). Let $X$ be a reflexive Banach space in the framework of Theorem 5.3(ii). Assume in addition that the kernel well-posedness of (5.1) from Definition 5.6 is satisfied at $(\bar{p}, \bar{x})$. Then all the conclusions of Theorem 5.3(ii) hold in the infinite-dimensional setting under consideration.

Proof. Let us show that the solution map (5.3) is $P S N C$ at the reference point $(\bar{p}, \bar{x})$ under the assumptions made. This is the only property needed to be checked to justify the conclusions of this theorem due to the results of Theorem 2.1 and the proof of Theorem 5.3(ii). 
To verify the PSNC property of $S$ at $(\bar{p}, \bar{x})$ according to its definition in (2.10), take sequences $\left(p_{k}, x_{k}\right) \rightarrow(\bar{p}, \bar{x})$ as $k \rightarrow \infty$ with $\left(p_{k}, x_{k}\right) \in \operatorname{gph} S$ for all $k \in \mathbb{N}$ and

$$
\left(p_{k}^{*}, x_{k}^{*}\right) \in \widehat{N}\left(\left(p_{k}, x_{k}\right) ; \operatorname{gph} S\right) \text { with } p_{k}^{*} \stackrel{w}{\rightarrow} 0 \text { and }\left\|x_{k}^{*}\right\| \rightarrow 0 \text { as } k \rightarrow \infty \text {. }
$$

Recall that the graph of $S$ has the inverse image representation (5.5), where the mapping $g: Z \times X \rightrightarrows X \times X^{*}$ defined in (5.4) has the surjective derivative at $(\bar{p}, \bar{x})$. Similarly to the proof of Lemma 5.1 by using [15, Lemma 1.16], we find sequences $\left(u_{k}, v_{k}\right) \rightarrow(\bar{x},-f(\bar{p}, \bar{x}))$ with $\left(u_{k}, v_{k}\right) \in \operatorname{gph} \mathcal{F}$ for all $k \in \mathbb{N}$ and

$$
\left\{\begin{array}{l}
\left(\widetilde{p}_{k}^{*}, \widetilde{x}_{k}^{*}\right) \in \nabla g(\bar{p}, \bar{x})^{*} \widehat{N}\left(\left(u_{k}, v_{k}\right) ; \operatorname{gph} \mathcal{F}\right) \text { with } \\
\left\|\widetilde{p}_{k}^{*}-p_{k}^{*}\right\| \rightarrow 0 \text { and }\left\|\widetilde{x}_{k}^{*}-x_{k}^{*}\right\| \rightarrow 0 \text { as } k \rightarrow \infty
\end{array}\right.
$$

It is easy to see from (5.22), (5.23), and the structure of $g$ in (5.4) that there are prenormals

$$
\left(u_{k}^{*}, v_{k}^{*}\right) \in \widehat{N}\left(\left(u_{k}, v_{k}\right) ; \operatorname{gph} \mathcal{F}\right) \text { for all } k \in \mathbb{N}
$$

satisfying the following relationships with $\left(\widetilde{p}_{k}^{*}, \widetilde{x}_{k}^{*}\right)$ in $(5.23)$ :

$$
\widetilde{p}_{k}^{*}=-\nabla_{p} f(\bar{p}, \bar{x})^{*} v_{k}^{*} \text { and } \widetilde{x}_{k}^{*}=u_{k}^{*}-\nabla_{x} f(\bar{p}, \bar{x})^{*} v_{k}^{*}
$$

Proceed now as in the proof of Theorem 4.1 for the prenormals (5.24) under consideration and define the active indices subsets $P \subset Q \subset I(\bar{x})$ as in (4.6) and (4.8), respectively, where $\lambda_{i k} \geq 0$ are determined from the representation

$$
u_{k}^{*}=\sum_{i \in Q} \lambda_{i k} x_{i}^{*}
$$

via the generating elements $\left\{x_{i}^{*} \mid i \in Q\right\}$ of the convex polyhedron (3.1). Then, as in the proof of Theorem 4.1, we get from (5.24) and (5.25) the inclusions

$$
\widetilde{x}_{k}^{*}+\nabla_{x} f(\bar{p}, \bar{x})^{*} v_{k}^{*} \in A_{Q, P} \text { and } v_{k}^{*} \in B_{Q, P}, \quad k \in \mathbb{N} .
$$

It is easy to conclude by the standard contradiction arguments based on the linear independence assumption on the active generating elements $\left\{x_{i}^{*} \mid i \in I(\bar{x})\right\}$ that the sequences $\left\{\lambda_{i k}\right\}$ are bounded for all $\in Q$. Thus we get without loss of generality that $\lambda_{i k} \rightarrow \lambda_{i} \geq 0$ as $k \rightarrow \infty$ whenever $i \in Q$. It follows from the convergence $\widetilde{p}_{k}^{*} \stackrel{w}{\rightarrow} 0$ due to (5.22) and (5.23) and the surjectivity of $\nabla_{p} f(\bar{p}, \bar{x})$ that $v_{k}^{*} \stackrel{w}{\rightarrow} 0$ as $k \rightarrow \infty$ by the first equality in (5.25). Observe further that $u_{k}^{*} \stackrel{w}{\rightarrow} 0$ as $k \rightarrow \infty$ by the second equality in (5.25). Now passing to the limit in (5.26) as $k \rightarrow \infty$, we arrive at $\sum_{i \in Q} \lambda_{i} x_{i}^{*}=0$, which implies that $\lambda_{i}=0$ for all $i \in Q$ by the linear independence of $\left\{x_{i}^{*} \mid i \in Q\right\}$. This gives

$$
\left\|\widetilde{x}_{k}^{*}+\nabla_{x} f(\bar{p}, \bar{x})^{*} v_{k}^{*}\right\| \rightarrow 0 \text { and hence }\left\|\nabla_{x} f(\bar{p}, \bar{x})^{*} v_{k}^{*}\right\| \rightarrow 0 \text { as } k \rightarrow \infty .
$$

Further, it follows from the construction of $B_{Q, P}$ in (3.15) and the set monotonicity property in (5.16) that the second inclusion in (5.27) can be replaced by

$$
v_{k}^{*} \in B_{J, J}=\operatorname{ker}\left\{x_{i}^{*} \mid i \in J\left(\bar{x}, \bar{x}^{*}\right)\right\}, \quad k \in \mathbb{N}
$$


where the equality in (5.29) is a direct consequence of the definitions. We can also easily observe that property (5.29) together with (5.28) and the kernel well-posedness of (5.1) at $(\bar{p}, \bar{x})$ yield that $\left\|v_{k}^{*}\right\| \rightarrow 0$ and hence $\left\|\tilde{p}_{k}^{*}\right\| \rightarrow 0$ as $k \rightarrow \infty$ by (5.25). Taking now (5.23) into account, conclude that the relationships in (5.22) imply that $\left\|p_{k}^{*}\right\| \rightarrow 0$ as $k \rightarrow \infty$, which justifies the PSNC property of $S$ at $(\bar{p}, \bar{x})$ and completes the proof of the theorem.

Finally, we present explicitly verifiable conditions, which simultaneously ensure the fulfillment of the coderivative criterion (5.13) in Theorem 5.3(ii) and the kernel well-posedness property of (5.1) from Definition 5.6 and thus efficiently describe important classes of variational inequalities that exhibit robust stability in finite and infinite dimensions.

Corollary 5.9 (robust stability under coercivity). Let $(\bar{p}, \bar{x}) \in \operatorname{gph} S$ for the solution map (5.3) to (5.1) with the reflexive spaces $X$ and $Z$ and with the linearly independent generating elements $\left\{x_{i}^{*} \mid i \in I(\bar{x})\right\}$. Assume that $f$ in (5.1) is strictly differentiable at $(\bar{p}, \bar{x})$, that $I(\bar{x})=J\left(\bar{x}, \bar{x}^{*}\right)$ with $\bar{x}^{*}=-f(\bar{p}, \bar{x})$, and that the operator $\nabla_{p} f(\bar{p}, \bar{x})$ is surjective. In addition we impose the conditions:

(a) the kernel well-posedness of (5.1) holds at $(\bar{p}, \bar{x})$,

(b) the operator $\nabla_{x} f(\bar{p}, \bar{x})$ is coercive on the kernel subspace $\operatorname{ker}\left\{x_{i}^{*} \mid i \in I(\bar{x})\right\}$,

which both are satisfied when $\nabla_{x} f(\bar{p}, \bar{x})$ is coercive on $X$. Then the solution map $S$ is Lipschitz-like around $(\bar{p}, \bar{x})$.

Proof. We show first that the imposed coercivity of $\nabla_{x} f(\bar{p}, \bar{x})$ on the kernel subspace implies the coderivative criterion (5.13). Observe that

$$
\operatorname{ker}\left\{x_{i}^{*} \mid i \in I(\bar{x})\right\}=B_{J, J}=B_{I, I}
$$

under the assumptions made and that the coderivative criterion (5.13) reads:

$$
\left[-\nabla_{x} f(\bar{p}, \bar{x})^{*} u \in A_{I, I}, \quad-u \in B_{I, I}\right] \Longrightarrow u=0 .
$$

It easily follows from the definitions of $A_{I, I}$ in (3.14) and the representation of $B_{I, I}$ in (5.30) that criterion (5.31) amounts to verify that

$$
\left[\nabla_{x} f(\bar{p}, \bar{x})^{*} u \in \operatorname{span}\left\{x_{i}^{*} \mid i \in I(\bar{x})\right\} \text { and } u \in \operatorname{ker}\left\{x_{i}^{*} \mid i \in I(\bar{x})\right\}\right] \Longrightarrow u=0 .
$$

Employing now the kernel coercivity (b) of the operator $A=\nabla_{x} f(\bar{p}, \bar{x})$ as in (5.19) with $L:=\operatorname{ker}\left\{x_{i}^{*} \mid i \in I(\bar{x})\right\}$ and using the above representations of $A_{I, I}$ and $B_{I, I}$ as well as the reflexivity of $X$, we find a constant $\mu>0$ such that

$$
\mu\|u\|^{2} \leq\left\langle\nabla_{x} f(\bar{p}, \bar{x}) u, u\right\rangle=\left\langle\nabla_{x} f(\bar{p}, \bar{x})^{*} u, u\right\rangle=0
$$

for any $u$ satisfying the inclusions in (5.32). The latter yields $u=0$ justifying implication (5.32). Thus the Lipschitz-like property of the solution map (5.3) follows, under the assumptions made in the corollary, from Theorem 5.8. To complete the proof, it remains to observe that the coercivity of $\nabla_{x} f(\bar{p}, \bar{x})$ on the whole space $X$ obviously implies (b) and ensures condition (a) of the corollary due to Proposition 5.7(c).

Our concluding remarks compare the stability results obtained in this section with those known in the literature. We also discuss some further extensions. 
Remark 5.10 (comparison with known results on robust stability). The results on robust stability of polyhedral variational inequalities most close to our study are obtained in $[3,28]$ in the case of finite-dimensional spaces of decision and parameter variables, with no evaluation of the exact Lipschitzian bound. Applications to robust stability in both papers $[3,28]$ are based on the coderivative characterization of the Lipschitz-like/Aubin property from Theorem 2.1 and coderivative calculations discussed above in Remark 4.7. In fact, paper [3] addresses the case of so-called canonical perturbations in polyhedral variational inequalities, which are linear with respect to the major parameter variable. The critical face characterization of robust stability established therein involves closed faces of some polyhedral critical cone built upon the tangent cone to the convex polyhedron $\Theta$. This characterization cannot be easily checked in general settings. It is worth emphasizing that results of [3] establishes the equivalence of the Lipschitz-like/Aubin property of solution maps to canonically perturbed variational inequality over convex polyhedra in finite dimensions to their strong regularity in Robinson's sense [24], which postulates locally single-valued Lipschitzian behavior.

Certain simplifications of the latter characterization is obtained in [28] on the base of the coderivative calculations from [27]. However, the robust stability conditions obtained in [28] also involve closed faces of some polyhedral cone associated with the tangent cone to the initial convex polyhedron $\Theta$.

Observe that our stability results are fully explicit and are expressed exclusively in terms of the initial data of the convex polyhedron $\Theta$ and the base mapping $f$ of the variational inequality (5.1) in both finite-dimensional and infinite-dimensional spaces. Since, in the finite-dimensional setting of [3], the Lipschitz-like property of solution maps is equivalent to Robinson's strong regularity, our explicit conditions provide also criteria for strong regularity of polyhedral variational inequalities in finite dimensions. It is a challenging open question whether this holds in infinite-dimensional spaces.

Remark 5.11 (further extensions). Combining coderivative calculations of Section 4 with coderivative formulas (mainly upper estimates) and PSNC conditions established in [15, Section 4.4] for solution maps to parametric generalized equations, we can obtain sufficient conditions for robust stability constructively expressed via the initial data of polyhedral variational inequalities (5.1) in both finite and infinite dimensions in a number of settings when the base mappings $f$ in (5.1) are nonsmooth or have nonsurjective derivatives.

Note finally that, employing the techniques developed in this paper together with those from [6] based on the transformation formula derived in [18], we can extend the robust stability results obtained here to variational inequalities over nonpolyhedral sets described by finitely many nonlinear inequality constraints. These and related topics will be considered in detail in our subsequent research.

\section{References}

[1] D. BARTL, A short algebraic proof of the Farkas lemma, SIAM J. Optim., 19 (2008), pp. 234-239. 
[2] J. M. Borwein And Q. J. ZHU, Techniques of Variational Analysis, Springer, New York, 2005.

[3] A. L. Dontchev and R. T. RockafellaR, Characterization of strong regularity for variational inequalities over polyhedral convex sets, SIAM J. Optim., 7 (1996), pp. $1087-1105$.

[4] F. FACChINEI AND J.-P. PANG, Finite-Dimensional Variational Inequalities and Complementarity Problems, Springer, New York, 2003.

[5] W. Geremew, B. S. Mordukhovich AND N. M. NAM, Coderivative calculus and metric regularity for constraint and variational systems, Nonlinear Anal., 70 (2009), pp. 529-552.

[6] R. Henrion, J. V. Outrata and T. Surowiec, On the coderivative of normal cone mappings to inequality systems, Nonlinear Anal., to appear.

[7] R. HENRION AND W. RömISCH, On M-stationary points for a stochastic equilibrium problem under equilibrium constraints in electricity spot market modeling, Appl. Math., 52 (2007), pp. 473-494.

[8] A. D. IofFE, Metric regularity and subdifferential calculus, Russian Math. Surv., 55 (2000), pp. 501-558.

[9] D. Klatte And B. Kummer, Nonsmooth Equations in Optimization. Regularity, Calculus, Methods and Applications, Kluwer, Dordrecht, 2002.

[10] A. B. LeVy AND B. S. MoRduKhovich, Coderivatives in parametric optimization, Math. Prog., 99 (2004), pp. 311-327.

[11] B. S. MoRduKHovich. Maximum principle in problems of time optimal control with nonsmooth constgraints, Appl. Math. Mech., 40 (1976), pp. 960-969.

[12] B. S. MoRdUKHOVICH, Metric approximations and necessary optimality conditions for general classes of extremal problems, Soviet Math. Dokl., 22 (1980), pp. 526-530.

[13] B. S. MordUKHOVICH, Sensitivity analysis in nonsmooth optimization, in Theoretical Aspects of Industrial Design, D. A. Field and V. Komkov (eds.), Proceedings in Applied Mathematics, Vol. 58, SIAM, Philadelphia, 1992, pp. 32-46.

[14] B. S. MoRdukHovich, Complete characterizations of openness, metric regularity and Lipschitzian properties of multifunctions, Trans. Amer. Math. Soc., 340 (1993), pp. $1-35$.

[15] B. S. MordukHovich, Variational Analysis and Generalized Differentiation, I: Basic Theory, Springer, Berlin, 2006.

[16] B. S. MordukHovich, Variational Analysis and Generalized Differentiation, II: Applications, Springer, Berlin, 2006. 
[17] B. S. MORDUKHOVICH AND J. V. OUTRATA, On second-order subdifferentials and their applications, SIAM J. Optim., 12 (2001), pp. 139-169.

[18] B. S. Mordukhovich and J. V. OUtrata, Coderivative analysis of quasi-variational inequalities with applications to stability and optimization, SIAM J. Optim., 18 (2007), pp. 389-412.

[19] J. V. OUTRATA, Mathematical programs with equilibrium constraints: Theory and numerical methods, in Nonsmooth Mechanics of Solids, CISM Lecture Notes, Vol. 485, J. Haslinger and G. E. Stavroulakis (eds.), Springer, New York, 2006, pp. 221-274.

[20] J. V. OUTRATA AND D. SUn, On the coderivative of the projection operator onto the second-order cone, Set-Valued Anal., 16 (2008), pp. 999-1014.

[21] R. A. Poliquin And R. T. Rockafellar, Tilt stability of a local minimum, SIAM J. Optim., 8 (1998), pp. 287-299.

[22] S. Ramaswamy, The Lax-Milgram theorem for Banach spaces, Proc. Japan Acad., 56 (1980), pp. 462-464.

[23] S. M. Robinson, Generalized equations and their solutions. Part 1: Basic theory, Math. Prog. Study, 10 (1979), pp. 128-141.

[24] S. M. Robinson, Strongly regular generalized equations, Math. Oper. Res., 5 (1980), pp. $43-62$.

[25] R. T. Rockafellar and R. J-B Wets, Variational Analysis, Springer, Berlin, 1998.

[26] W. SchirotzeK, Nonsmooth Analysis, Springer, Berlin, 2007.

[27] J.-C. YAO AND N. D. YEN, Coderivative calculation related to a parametric affine variational inequality. Part 1: Basic calculations, Acta Math. Vietnamica, to appear.

[28] J.-C. YAO AND N. D. YEN, Pointbased calculation related to a parametric affine variational inequality. Part 2: Applications, Pacific J. Optim., to appear.

[29] J. J. YE, Constraint qualifications and necessary optimality conditions for optimization problems with variational inequality constraints, SIAM J. Optim., 10 (2000), pp. 943962. 